\title{
Habitable Moist Atmospheres on Terrestrial Planets near the Inner Edge of the Habitable Zone around M Dwarfs
}

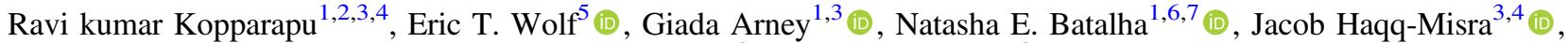 \\ Simon L. Grimm ${ }^{8}$ (D), and Kevin Heng ${ }^{8}$ (D) \\ ${ }^{1}$ NASA Goddard Space Flight Center, 8800 Greenbelt Rd, Mail Stop 699.0 Building 34, Greenbelt, MD 20771, USA \\ ${ }^{2}$ Department of Astronomy, University of Maryland College Park, College Park, MD, USA \\ ${ }^{3}$ NASA Astrobiology Institute's Virtual Planetary Laboratory, P.O. Box 351580, Seattle, WA 98195, USA \\ ${ }^{4}$ Blue Marble Space Institute of Science, 1001 4th Ave., Suite 3201, Seattle, WA 98154, USA \\ ${ }^{5}$ Department of Atmospheric and Oceanic Sciences, Laboratory for Atmospheric and Space Physics, University of Colorado Boulder, Boulder, CO 80309, USA \\ ${ }^{6}$ Department of Astronomy \& Astrophysics, Penn State University, Davey Laboratory, University Park, PA 16802, USA \\ ${ }^{7}$ Center for Exoplanets and Habitable Worlds, The Pennsylvania State University, University Park, PA 16802, USA \\ ${ }^{8}$ Center for Space Habitability, University of Bern, Sidlerstrasse 5, Exact Sciences Building, CH-3012, Bern, Switzerland \\ Received 2017 March 19; revised 2017 June 6; accepted 2017 June 28; published 2017 August 4
}

\begin{abstract}
Terrestrial planets in the habitable zones (HZs) of low-mass stars and cool dwarfs have received significant scrutiny recently. Transit spectroscopy of such planets with the James Webb Space Telescope (JWST) represents our best shot at obtaining the spectrum of a habitable planet within the next decade. As these planets are likely tidally locked, improved 3D numerical simulations of such planetary atmospheres are needed to guide target selection. Here we use a 3D climate system model, updated with new water-vapor absorption coefficients derived from the HITRAN 2012 database, to study ocean-covered planets at the inner edge of the HZ around late M to mid$\mathrm{K}$ stars $\left(2600 \mathrm{~K} \leqslant T_{\text {eff }} \leqslant 4500 \mathrm{~K}\right)$. Our results indicate that these updated water-vapor coefficients result in significant warming compared to previous studies, so the inner HZ around M dwarfs is not as close as suggested by earlier work. Assuming synchronously rotating Earth-sized and Earth-mass planets with background 1 bar $\mathrm{N}_{2}$ atmospheres, we find that planets at the inner $\mathrm{HZ}$ of stars with $T_{\text {eff }}>3000 \mathrm{~K}$ undergo the classical "moist greenhouse" $\left(\mathrm{H}_{2} \mathrm{O}\right.$ mixing ratio $>10^{-3}$ in the stratosphere $)$ at significantly lower surface temperature $(\sim 280 \mathrm{~K})$ in our 3D model compared with 1D climate models $(\sim 340 \mathrm{~K})$. This implies that some planets around low-mass stars can simultaneously undergo water loss and remain habitable. However, for stars with $T_{\text {eff }} \leqslant 3000 \mathrm{~K}$, planets at the inner HZ may directly transition to a runaway state, while bypassing the moist greenhouse water loss entirely. We analyze transmission spectra of planets in a moist greenhouse regime and find that there are several prominent $\mathrm{H}_{2} \mathrm{O}$ features, including a broad feature between 5 and $8 \mu \mathrm{m}$, within JWST MIRI instrument range. Thus, relying only on standard Earth-analog spectra with $24 \mathrm{hr}$ rotation period around $\mathrm{M}$ dwarfs for habitability studies will miss the strong $\mathrm{H}_{2} \mathrm{O}$ features that one would expect to see on synchronously rotating planets around $\mathrm{M}$ dwarf stars, with JWST.
\end{abstract}

Key words: planets and satellites: atmospheres - planets and satellites: composition - planets and satellites: detection - planets and satellites: terrestrial planets - stars: low-mass

\section{Introduction}

Discoveries of terrestrial planets in the habitable zone (HZ) by both the Kepler mission and ground-based surveys (Anglada-Escudé et al. 2016; Kane et al. 2016; Gillon et al. 2017) have shown that potentially habitable planets are common in our Galaxy (Petigura et al. 2013; Foreman-Mackey et al. 2014; Dressing \& Charbonneau 2015; Silburt et al. 2015). With the discovery of the nearest $\mathrm{HZ}$ planets around Proxima Centauri and TRAPPIST-1, we also have an opportunity to characterize the atmospheres of these planets in the not-toodistant future. There is an extensive literature on modeling and speculating on the potential habitability of terrestrial planets in the HZ (Selsis et al. 2007; Wordsworth et al. 2010; Abe et al. 2011; Haqq-Misra \& Kopparapu 2015; Barnes et al. 2016; Meadows et al. 2016; Ribas et al. 2016; Turbet et al. 2016; Bolmont et al. 2017), and studies such as these provide a valuable contribution in understanding, and eventually interpreting, any biosignatures. In particular, low-mass stars (late $\mathrm{K}$ and $\mathrm{M}$ dwarf stars) provide our best opportunity for detecting and characterizing habitable terrestrial planets in the coming decade. The small size and compact HZs of these stars allow for a greater chance of detection of terrestrial-sized planets, and the higher frequency of transits for planets in the HZs of lowmass stars (due to their shorter orbital periods) means that higher signal-to-noise levels can be observed in less time compared to planets orbiting around hotter stars.

Crucial to interpreting these discoveries is the location of the $\mathrm{HZ}$, where liquid water remains thermodynamically stable on the planet's surface indefinitely. Note that this particular definition of the $\mathrm{HZ}$ is geared toward remote detection of life through observations of exoplanet atmospheres, and not a statement on the planets' habitability itself (Kasting et al. 2014). It is also useful in determining the occurrence of potentially habitable planets in our galaxy, as was done with Kepler data. Several groups have studied the limits of the mainsequence HZ (Kasting et al. 1993; Pierrehumbert \& Gaidos 2011; Kopparapu et al. 2013, 2014, 2016; Leconte et al. 2013b; Yang et al. 2013, 2014a; Zsom et al. 2013; Wolf \& Toon 2014; Godolt et al. 2015; Leconte et al. 2015; Way et al. 2015; Wolf \& Toon 2015; Haqq-Misra et al. 2016; Ramirez \& Kaltengger 2017; Salameh et al. 2017) using both 1D and 3D climate models and corresponding climate transitions that planets undergo at these limits. Many of these models assume water- 
rich $(\sim 1$ Earth-ocean) planets, which is reasonable if one wants to study the surface habitability of a planet. The validity of this assumption can be debated, particularly for planets in the $\mathrm{HZ}$ of M dwarf stars, due to their high pre-main-sequence luminosity (Ramirez \& Kaltengger 2014; Luger \& Barnes 2015; Tian \& Ida 2015), or atmospheric erosion due to high X-ray and UV fluxes (Airapetian et al. 2017). But this can only be verified with more observations, and more detections, of these kinds of planets.

Both 1D and 3D models show that, for a water-rich planet near the inner edge of the HZ, as the surface temperature increases owing to increased stellar radiation, water vapor becomes a significant fraction of both the troposphere and stratosphere (Kasting et al. 2015). $\mathrm{H}_{2} \mathrm{O}$ is a strong absorber in both the long wave and the short wave. Thus, increasing atmospheric water vapor limits the maximum outgoing thermal radiation (Goldblatt et al. 2013) and also increases absorption of solar radiation in the near-IR, thereby lowering a planet's albedo. This effect is accentuated on planets around $\mathrm{M}$ dwarfs because the radiation from $M$ dwarfs peaks in the near-IR, where there are strong water absorption bands. Our own previous work on HZs (Kopparapu et al. 2013, 2014) using a $1 \mathrm{D}$ radiative-convective, cloud-free climate model, and updated with new and stronger $\mathrm{H}_{2} \mathrm{O}$ absorption coefficients from HITRAN2008 and HITEMP2010 line-by-line (LBL) databases, showed that the inner edge of the $\mathrm{HZ}$ can be significantly farther from the star than previous estimates (Kasting et al. 1993).

However, a critical component for discerning the climate of terrestrial planets around $\mathrm{M}$ dwarf stars, and thus the HZ, is to consider the effects of slow and synchronous rotation caused by tidal locking. Planets in the HZ of low-mass stars are likely to find themselves in synchronous rotation owing to strong tidal interactions with the host star (Dobrovolskis 2009; Barnes et al. 2013). Planets in synchronous rotation will have permanent day and night hemispheres (Joshi et al. 1997). Slow planetary rotation has a profound impact on the climate of Earth-like planets (Merlis \& Schneider 2010; Yang et al. 2013, 2014; Carone et al. 2014, 2015, 2016; Way et al. 2016). Slow rotation weakens the Coriolis effect and causes the atmospheric circulation to shift from a "rapidly rotating" regime, characterized by zonal uniformity (banded cloud formation) and symmetry about the equator (e.g., like Earth currently), to a "slowly rotating" regime, characterized by dayside to nightside heat (energy) transport and circular symmetry about the substellar point. The transition between these circulation regimes depends on the radius of the planet and rotation rate through the Rossby radius of deformation (the Rossby radius of deformation is the length scale at which the Coriolis effect becomes important for determining the motion of an air parcel). For an Earth-sized planet, the Rossby radius of deformation exceeds the planet size when the rotational period reaches $\sim 5$ Earth days (Yang et al. 2014). The climates of these slowrotating worlds are strongly influenced by the circulation regime, as convection, clouds, and horizontal transport are fundamentally altered (Yang et al. 2013; Kopparapu et al. 2016). On slow-rotating planets, thick clouds at the substellar point tend to cool the planet by increasing the albedo. Thus, any attempt to accurately simulate the climate of planets around low-mass stars must take into account these inherently 3D effects, brought about by the slow and synchronous rotation of the planet. These effects cannot be meaningfully parameterized in 1D models used for studying habitable extrasolar planets (e.g., Kopparapu et al. 2013; Meadows et al. 2016).

Furthermore, recent three-dimensional climate modeling studies predict that rapidly rotating Earth-like planets undergo a sharp transition between temperate and moist greenhouse climate states (Wolf \& Toon 2015; Popp et al. 2016). However, another study with a different model (Leconte et al. 2013b) found that cold stratosphere appears to preclude an increase of stratospheric humidity, preventing a moist greenhouse state. Wolf \& Toon (2015) used a modified version of the NCAR CAM4 3D climate model and found that stable, moist greenhouse solutions are indeed possible for an Earth-Sun configuration. Furthermore, Wolf \& Toon (2015) found comparable stratospheric temperatures (always $\geqslant 150 \mathrm{~K}$ ) to Leconte et al. (2013b) at low surface temperatures, but at high surface temperatures they found much warmer (up to $\sim 210 \mathrm{~K}$ ) stratospheric temperatures and correspondingly higher stratospheric $\mathrm{H}_{2} \mathrm{O}$ mixing ratios. In a third study, Popp et al. (2016) used the ECHAM6 3D climate model and found stable moist greenhouse solutions very similar in description to those found by Wolf \& Toon (2015), albeit at lower stellar fluxes in their model. If the results of Popp et al. (2016) and Wolf \& Toon (2015) are correct, then water would be lost from a planet's surface well before a runaway greenhouse occurs, and possibly before surface temperatures can rise above limits for habitability. Another study by Kasting et al. (2015), using a 1D climate model, found that at higher surface temperatures the stratosphere warms and water vapor indeed becomes a dominant component gas in the atmosphere. Thus, contrary to Leconte et al. (2013b), catastrophic water loss may be possible from a moist greenhouse planet.

Wolf \& Toon (2015) argue that the transition to a moist greenhouse is associated with a fundamental change to the radiative-convective state of the atmosphere. For a rapidly rotating Earth-like planet around the Sun, if global mean temperatures exceed $\sim 310 \mathrm{~K}$, then increasing water vapor causes both strong solar absorption and inefficient radiative cooling in the low atmosphere, resulting in net radiative heating of the layers above the surface. This heating forms a thermal inversion, which is stable against deep convection and thus prohibits the formation of convective clouds (See Section 3.3 for more discussion).

Kopparapu et al. (2016) found that the above-described radiative-convective transition also occurs on slow and synchronously rotating Earth-like planets, which are expected around low-mass stars. While rapidly rotating planets can maintain climatological stability beyond this transition owing to cloud adjustments in the upper atmosphere, this transition is catastrophic for planets located near the inner edge of the $\mathrm{HZ}$ around low-mass stars. Slow-rotating planets are effectively shielded from the host star by thick convectively produced clouds located around the substellar point. These planets can remain habitable despite incident stellar fluxes up to twice that of the present-day Earth (Yang et al. 2014; Kopparapu et al. 2016; Way et al. 2016). However, the radiative-convective transition and subsequent onset of the near surface inversion stabilizes the substellar atmosphere, and thus the convective cloud deck rapidly dissipates. Even a small dent in this substellar cloud shield then lets in a tremendous amount of solar radiation, destabilizing climate toward an immediate thermal runaway. 
Here, our goal is to study terrestrial planet atmospheres near the inner edge of the HZ around low-mass stars $(2600 \mathrm{~K}$ $\leqslant T_{\text {eff }} \leqslant 4500 \mathrm{~K}$ ), using a state-of-the-art 3D climate system model, with updated and validated $\mathrm{H}_{2} \mathrm{O}$ radiative transfer. We provide new estimates on the inner edge of the $\mathrm{HZ}$ for lowmass stars, while differentiating between moist greenhouse and runaway greenhouse limits to planetary habitability. Our results indicate that, even for synchronously rotating planets, the inner edge of the $\mathrm{HZ}$ around low-mass stars is significantly farther out (i.e., at lower stellar fluxes) than is predicted by earlier studies (Yang et al. 2013; Kopparapu et al. 2016).

Even more interesting is that several simulated planets in the slow rotation regime have water-rich atmospheres (mixing ratios $\sim>10^{-3}$ ) that are within the classic "moist greenhouse" regime, but maintain mild surface temperatures $(\sim 280 \mathrm{~K})$ owing to the thick cloud shield near the substellar point. In comparison, a moist greenhouse in a 1D climate model occurs at a surface temperature of $\sim 340 \mathrm{~K}$, and $3 \mathrm{D}$ climate models show moist greenhouse atmospheres occurring with surface temperatures of $\sim 350 \mathrm{~K}$ (Wolf \& Toon 2015; Popp et al. 2016), in each case too hot for life similar to ours (Sherwood \& Huber 2010). Here, we calculate that these water-rich habitable atmospheres are stable for hundreds of million or even several billion years. Depending on the amount of water lost, it is possible that some planets can smoothly transition to waterpoor or dry planets and can still retain habitability in these dry states (Abe et al. 2011; Leconte et al. 2013a; Kodama et al. 2015). We speculate on the number of such planets that can be detected by the TESS mission and perhaps eventually be characterized by JWST, as these planets are much closer to the star than planets within the conventional HZ.

The outline of the paper is as follows: In Section 2 we describe the updates to our 3D General Circulation Model (GCM) climate model. In Section 3 we present results. In Section 4 we discuss the ramifications of our results on habitable zones and observations, and we conclude in Section 5 .

\section{Model}

\subsection{Atmospheric Model}

Here, we use a modified version of the Community Atmosphere Model version 4 (CAM4) from the National Center for Atmospheric Research in Boulder, Colorado (Neale et al. 2010). We use $4^{\circ} \times 5^{\circ}$ horizontal resolution, with 40 verticals levels extending to a model top of $\sim 1$ mbar. For all simulations we assume a planet with Earth radius, mass, and gravity. The atmosphere is taken to consist only of $\mathrm{N}_{2}$ and $\mathrm{H}_{2} \mathrm{O}$. Other species such as $\mathrm{O}_{2}, \mathrm{O}_{3}, \mathrm{CO}_{2}, \mathrm{CH}_{4}$, and trace gases have been removed for simplicity, and habitable planets with active carbonate-silicate cycles should have low $\mathrm{CO}_{2}$ levels if their surface temperatures are hot. Thus, here our calculation of the inner edge of the habitable zone represents an innermost boundary. The addition of other greenhouse gas species would serve only to push the inner edge of the HZ farther away from the star. The total pressure of the atmosphere is given as $P_{\text {tot }}=P_{\mathrm{H}_{2} \mathrm{O}}+P_{\mathrm{N}_{2}}$, where $P_{x}$ is the partial pressure of each gaseous component. $P_{\mathrm{N}_{2}}$ is set to 1 bar in all simulations, while atmospheric water vapor varies self-consistently depending on the temperature and relative humidity of the atmosphere.

We assume an aquaplanet (i.e., an entirely ocean-covered planet with no land), with a $50 \mathrm{~m}$ deep thermodynamic "slab" ocean (Bitz et al. 2012). Ocean heat transport is to set to zero everywhere. While we focus on the inner edge of the HZ, many of our simulations still have ice cover. We include sea ice using the Los Alamos sea-ice model CICE version 4 (Hunke \& Lipscomb 2008). We use the default albedo parameterization, which divides ocean, sea ice, and snow albedos into two bands: visible and near-IR divided at $0.7 \mu \mathrm{m}$. Following Shields et al. (2013), we set the visible (near-IR) sea-ice albedo to 0.67 (0.3), the snow albedo to $0.8(0.68)$, and the ocean albedo to 0.07 (0.06), respectively.

We use the finite-volume (FV) dynamical core (Lin \& Rood 1996). The FV dynamical core has been modified to improve numerical stability (courtesy of $\mathrm{C}$. Bardeen). This entails incrementally applying physics tendencies for temperature and wind speed evenly throughout the dynamical substeps. Note that several additional sensitivity tests were conducted using the spectral element dynamical core on a cubed-sphere grid (Lauritzen et al. 2014). We use physics time steps of 30 minutes with 48 dynamical substeps per physics time step. The radiative transfer is calculated once every 90 minutes. All simulations are initiated with surface temperatures matching that of the modern Earth. Simulations are run until they enter a runaway state or reach statistically steady state, which typically takes about 40 Earth years.

\subsection{Radiative Transfer}

Previous calculations of the inner edge of the HZ for tidally locked planets around late $\mathrm{K}$ and $\mathrm{M}$ dwarf stars were conducted with CAM3 and CAM4 (Yang et al. 2014; Wang et al. 2016; Kopparapu et al. 2016), using the native (i.e., unmodified) radiative transfer code. According to the scientific description of $\mathrm{CAM},{ }^{9}$ the long-wave radiative transfer is based on the broadband model approach described by Kiehl \& Briegleb and Kiehl \& Ramanathan. The short-wave radiative transfer uses the $\delta$-Eddington approximation described in Briegleb (1992), across a coarse pseudo-spectral grid. Absorption by water vapor is based on the HITRAN2000 line database (Rothman et al. 2003) and incorporates the Clough, Kneizys, and Davies (CKD) 2.4 prescription for the continuum (Clough et al. 1989). Recently it was shown that the native CAM radiative transfer code significantly underestimates absorption by water vapor in both the long wave and the short wave (Yang et al. 2016). This means that the inner edges of the habitable zone defined by Yang et al. (2014) and Kopparapu et al. (2016) are both placed too close to the host star.

Yang et al. (2016) also indicate that the radiative transfer code first described by Wolf \& Toon (2013) and later used by Wolf \& Toon $(2014,2015)$ to study moist greenhouse atmospheres for Earth around the Sun does well in the long wave against its reference model (LBLRTM), but absorbs too much solar radiation for warm $\left(T_{s}>300 \mathrm{~K}\right)$, saturated atmospheres. Another result to emerge from Yang et al. (2016) is that spectral resolution in the near-IR is critical for accurately calculating short-wave radiative transfer in waterrich atmospheres. Here, we improve on the radiative transfer scheme first described in Wolf \& Toon (2013), by implementing a new correlated- $k$ approximation using the latest spectral database, HITRAN 2012, and by increasing the spectral resolution in the near-IR. New $k$-coefficients were

\footnotetext{
9 http://www.cesm.ucar.edu/models/atm-cam/docs/description/ description.pdf
} 

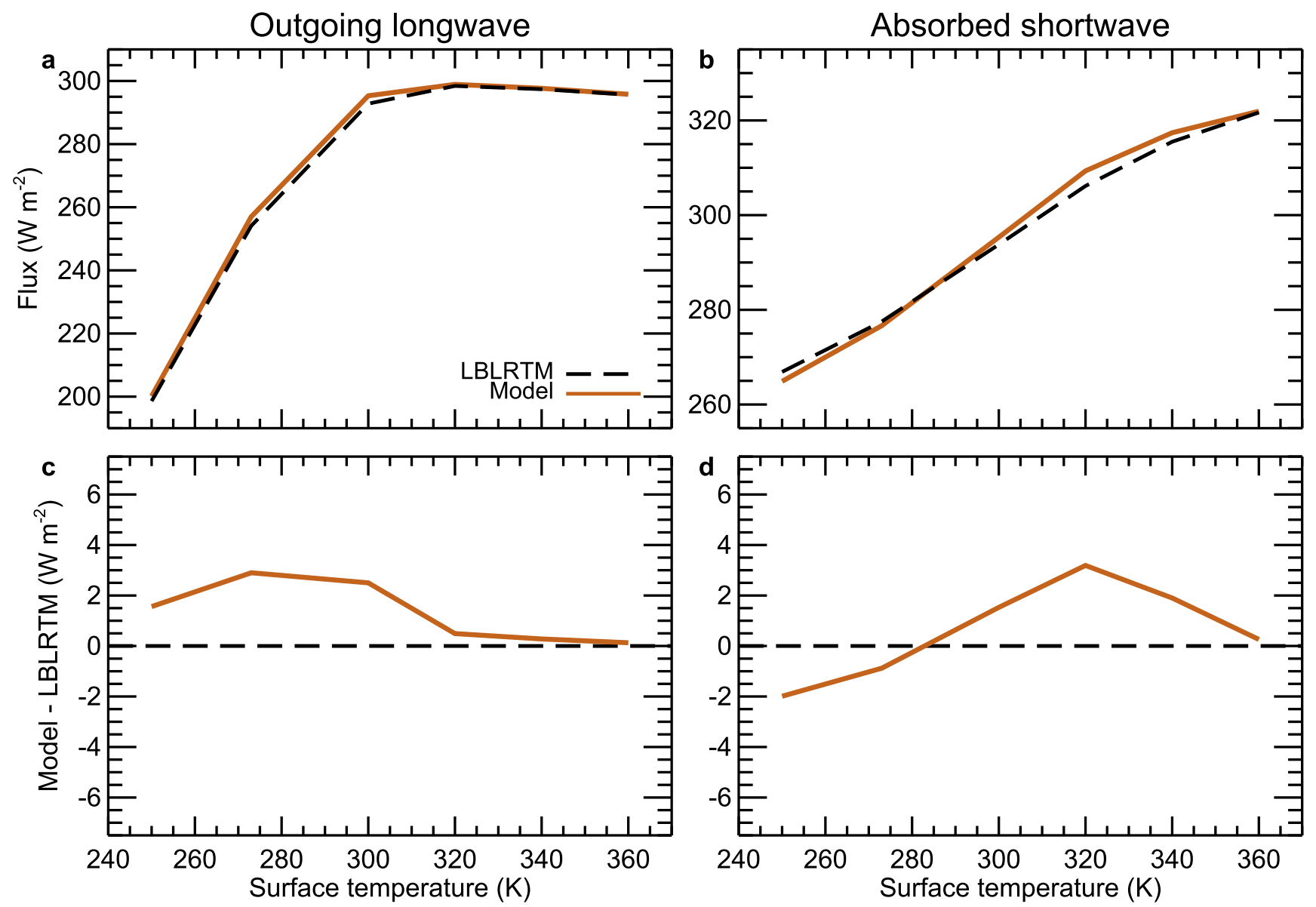

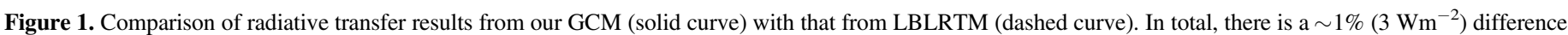
between the two codes in both long wave (left) and short wave (right).

created using HELIOS-K, a new open-source spectral sorting program designed for graphics processing units (Grimm \& Heng 2015). The utilization of massively parallel graphics processing units enables a several-order-of-magnitude increase in computational efficiency. This allowed us to test numerous different spectral resolutions and achieve an optimal balance of speed and accuracy for our project. We settled on a 42 spectral interval grid ranging from 10 to $50,000 \mathrm{~cm}^{-1}$, with 8 Gauss points per interval. The shortwave stream is computed over 35 spectral intervals from 820 to $50,000 \mathrm{~cm}^{-1}$. The long-wave stream is computed over 19 spectral intervals from 10 to $4000 \mathrm{~cm}^{-1}$.

We only consider absorption by $\mathrm{H}_{2} \mathrm{O}$, with the correlated $k$-coefficients calculated for a pressure grid from 10 bar to 0.01 mbar with successive pressure levels following $\log 10(\mathrm{P} 1 /$ $\mathrm{P} 2)=0.1$, and a temperature grid from 100 to $500 \mathrm{~K}$ with $\Delta T=25 \mathrm{~K}$. Furthermore, we have implemented a new watervapor continuum parameterization, following the formalism of Paynter \& Ramaswamy (2011). This continuum treatment is derived from laboratory measurements taken at temperatures appropriate for atmospheres expected near the inner edge of the habitable zone and is stronger than the commonly used MT-CKD continuum. Finally, we have implemented direct integration by midlayer Planck functions to correct some unphysical sawtoothing of heating rates found near the model top in previous versions. We use the two-stream radiative transfer solver from Toon et al. (1989).
Cloud overlap is treated using the Monte Carlo Independent Column Approximation (MCICA), assuming maximum-random overlap (Pincus et al. 2003). MCICA uses stochastically generated subcolumns to represent subgrid-scale cloud variability. Subcolumns are then randomly sampled across the spectral integration. MCICA is specifically designed for use in 3D models and is well tested in both GCMs (Barker et al. 2008) and numerical weather prediction models (Hill et al. 2015).

To test our code, we follow the methods of Yang et al. (2016), using clear-sky saturated atmospheric profiles with surface temperatures ranging from 250 to $360 \mathrm{~K}$, and $200 \mathrm{~K}$ isothermal stratospheres. For short-wave calculations we assume a $3400 \mathrm{~K}$ blackbody stellar spectrum as input. In Figure 1 we compare results from our correlated- $k$ radiative transfer code with that from a line-by-line code, LBLRTM (Clough et al. 2005), for both the long wave and short wave. Calculations of both outgoing long-wave radiation (OLR) and absorbed short-wave radiation (ASR) remain within $\sim 3 \mathrm{~W} \mathrm{~m}^{-2}$ compared with LBLRTM for all atmospheric profiles tested. This is accurate to within about $\sim 1 \%$ with respect to OLR and ASR. Accuracy is substantially improved compared to previous iterations of our correlated- $k$ code and also compared to the native CAM radiative transfer (Yang et al. 2016).

\subsection{Moist Physics}

Condensation, precipitation, and evaporation use the bulk microphysical parameterizations of Rasch \& Kristjánsson (1998). Deep convection is treated using the parameterization 
of Zhang \& McFarlane (1995). This scheme has been improved to include convective momentum transport and dilute entraining plumes (Raymond \& Blyth, 1986, 1992). The deep convection scheme uses a plume ensemble approach, where convective updrafts and downdrafts occur wherever the low atmosphere becomes conditionally unstable. The entropy closure calculation within the deep convection scheme has been updated (courtesy of C.A. Shields) to use the more robust numerical approach of Brent (1973). A secondary convective parameterization treats shallow convective overturning at all layers in the atmosphere (Hack 1994). CAM4 self-consistently accounts for changing atmospheric pressures due to changing water-vapor amounts in each grid cell. The total grid cell mass is determined from tendencies imparted by advection, convection, turbulent mixing, and large-scale stable condensation and evaporation. CAM4 uses virtual temperature corrections to account for variations in density, and a similar approach accounts for the variation in specific heat between moist and dry air.

Cloud fractions are determined for three separate types of clouds. Marine stratus cloud fractions depend on the stratification of the atmosphere between the surface and the 700 mbar level. Convective cloud fractions depend on the stability of the atmosphere and the convective mass flux. Layered clouds at all altitudes depend on the relative humidity and the pressure level. Cloud liquid droplet radii are assumed to be $14 \mu \mathrm{m}$ everywhere in the model. Ice cloud particle effective radii follow a temperature-dependent parameterization and can vary in size from a few tenths to a few hundred microns. A typical ice cloud in the model has an effective radius of $\sim 50 \mu \mathrm{m}$ at air temperatures of $240 \mathrm{~K}$ and $\sim 20 \mu \mathrm{m}$ at air temperatures of $200 \mathrm{~K}$.

\subsection{Stellar Properties}

We conduct simulations for planets around stars with effective stellar temperatures of 2600, 3000, 3300, 3700, 4000 , and $4500 \mathrm{~K}$. We assume that all of our planets are in synchronous rotation with their host stars. Leconte et al. (2015) argue that planets are expected to have a nonsynchronous rotation if they are in the habitable zone of stars more massive than $\sim 0.5$ to $0.7 M_{\odot}$ (depending on their location in the habitable zone). Our stellar properties remain below this limit. The orbital period and thus the planetary rotation rate of each case are calculated self-consistently with Kepler's laws, following Equation (3) in Kopparapu et al. (2016). The stellar effective temperature, mass, luminosity, stellar flux at the planet, and planetary rotation rate are strictly intertwined. We repeat the equation from Kopparapu et al. (2016), as this is critical in choosing our rotational periods of planets in our simulations:

$$
P(\text { years })=\left[\left(\frac{L_{\star} / L_{\odot}}{F_{P} / F_{\oplus}}\right)^{3 / 4}\right]\left[M_{\star} / M_{\odot}\right]^{-1 / 2},
$$

where $P$ is the orbital period of the planet (for synchronous rotation, it is also the rotational period), $L_{\star} / L_{\odot}$ is the luminosity of the star with respect to the bolometric luminosity of the Sun, $F_{P} / F_{\oplus}$ is the stellar flux incident on a planet $\left(F_{P}\right)$ compared to the flux on Earth $\left(F_{\oplus}\right)$, and $M_{\star} / M_{\odot}$ is the stellar mass in solar mass units. This equation indicates that the orbital period of a planet in synchronous rotation can be calculated from the luminosity, mass, and incident flux, and it cannot be chosen irrespective of the stellar type. We then use a parameterized relationship between $T_{\text {eff }}, M_{\star}$, and $L_{\star}$ (as shown in Table 1 of Kopparapu et al. 2016) to choose a specific stellar temperature. A list of simulations with stellar and planetary characteristics shown in Figures 3-5 is tabulated in Table 1.

For stars with $T_{\text {eff }}>3200 \mathrm{~K}$, we use the empirically derived relationships between the mass, luminosity, and effective temperature of the host stars from Boyajian et al. (2013). These relationships are valid for stellar metallicities ranging from $[\mathrm{Fe} / \mathrm{H}]=-0.5$ to +0.1 dex. Note that Boyajian et al. (2013) conclude that metallicity only affects the color index of a star; however, metallicity does not appear to impact the global properties of a star: temperature, radius, and luminosity. Stars with $T_{\text {eff }}<3200 \mathrm{~K}$ fall outside the validity range for the fits provided by Boyajian et al. (2013). Thus, in this regime we use the stellar properties from the Baraffe et al. (2002) stellar evolutionary models, assuming an age of $\sim 3 \mathrm{Gyr}(\log t$ $(y r)=9.50)$. For all cases, we use stellar spectra from the BTSETTL grid of models (Allard et al. 2003, 2007), assuming a stellar metallicity of $[\mathrm{Fe} / \mathrm{H}]=0.0$. We conduct simulations for planets under increasing stellar fluxes, until a runaway greenhouse is triggered.

\subsection{The SMART Radiative Transfer Model}

The Spectral Mapping Atmospheric Radiative Transfer Model (SMART) is a versatile line-by-line fully multiple scattering 1D radiative transfer model (Meadows \& Crisp 1996), capable of simulating a wide variety of planets. It has been validated against solar system objects (Arney et al. 2014; Robinson et al. 2014) and has been used to study a wide variety of types of atmospheres (Robinson et al. 2011; Arney et al. 2016; Schwieterman et al. 2016; Charnay et al. 2015). SMART has been modified to calculate transit spectra, including the effects of refraction (Misra et al. 2014). Transit spectra can be generated from GCM results by using atmospheric columns from the planetary terminator region.

\subsection{PandExo: A JWST Simulator}

We use PandExo, a publicly available simulator for JWST and HST. PandExo is described extensively in Batalha et al. 2017, but we briefly describe it here. PandExo relies on STScI's exposure time calculator Pandeia (Pontoppidan et al. 2016) to compute pixel-level simulations based on the most upto-date instrument characterization data. Therefore, it includes simulations of throughputs, background noise, read noise, point-spread functions, optical paths, and saturation levels. However, it does not fully simulate detector systematics, which have been suggested to create a noise floor of 20-30 ppm for the near-IR instruments and $50 \mathrm{ppm}$ for MIRI (Greene et al. 2016). It also does not simulate any time-varying noise sources such as stellar variability or spacecraft jitter and drift, which could add to JWST's systematic noise floor (Barstow et al. 2015).

\section{Results}

\subsection{Control Simulations}

First, we compare several sets of idealized simulations of synchronously rotating planets in order to determine the effect on climate of our updates to the radiative transfer code. In 
Table 1

Simulation Cases Shown in Figures 3-5

\begin{tabular}{|c|c|c|c|c|c|}
\hline$T_{\text {eff }}(\mathrm{K})$ & $L / L_{\odot}$ & $M / M_{\odot}$ & $F_{P} / F_{\oplus}$ & $P($ days $)$ & $\begin{array}{l}\text { Model Top } \mathrm{H}_{2} \mathrm{O} \\
\text { Mixing Ratio } \\
\quad(1 \text { mbar })\end{array}$ \\
\hline 4500 & 0.189 & 0.72 & $\begin{array}{l}1.323 \\
1.470 \\
1.617 \\
\\
1.654 \\
1.691 \\
1.727\end{array}$ & $\begin{array}{r}100.20 \\
92.59 \\
86.20\end{array}$ & $\begin{array}{c}2.97 \times 10^{-6} \\
7.04 \times 10^{-5} \\
1.41 \times 10^{-3} \\
(\text { water loss) } \\
7.41 \times 10^{-3} \\
1.79 \times 10^{-2} \\
\text { (runaway) }\end{array}$ \\
\hline 4000 & 0.0878 & 0.628 & $\begin{array}{l}1.176 \\
1.323 \\
1.397 \\
1.470 \\
\\
1.507\end{array}$ & $\begin{array}{l}65.79 \\
60.23 \\
57.83 \\
55.65 \\
\\
54.63\end{array}$ & $\begin{array}{c}1.76 \times 10^{-5} \\
3.61 \times 10^{-4} \\
1.48 \times 10^{-3} \\
1.64 \times 10^{-2} \\
\text { (water loss) } \\
\text { (runaway) }\end{array}$ \\
\hline 3700 & 0.0429 & 0.520 & $\begin{array}{l}1.102 \\
1.176 \\
1.323 \\
\\
1.397 \\
1.433\end{array}$ & $\begin{array}{l}44.33 \\
42.24 \\
38.66 \\
\\
37.13 \\
36.41\end{array}$ & $\begin{array}{c}2.19 \times 10^{-5} \\
7.85 \times 10^{-5} \\
2.89 \times 10^{-3} \\
\text { (water loss) } \\
2.82 \times 10^{-2} \\
\text { (runaway) }\end{array}$ \\
\hline 3300 & 0.00972 & 0.249 & $\begin{array}{l}1.029 \\
1.176 \\
1.213 \\
1.250 \\
\\
1.280\end{array}$ & $\begin{array}{l}22.12 \\
20.02 \\
19.57 \\
19.13 \\
\\
18.72\end{array}$ & $\begin{array}{c}3.41 \times 10^{-5} \\
1.24 \times 10^{-4} \\
5.55 \times 10^{-4} \\
7.60 \times 10^{-3} \\
\text { (water loss) } \\
\text { (runaway) }\end{array}$ \\
\hline 3000 & 0.00183 & 0.143 & $\begin{array}{l}0.955 \\
1.029 \\
1.103 \\
1.139 \\
1.158 \\
1.176\end{array}$ & $\begin{array}{l}8.83 \\
8.35 \\
7.93 \\
7.74 \\
7.65 \\
7.56\end{array}$ & $\begin{array}{c}4.14 \times 10^{-5} \\
2.12 \times 10^{-4} \\
2.57 \times 10^{-4} \\
5.14 \times 10^{-4} \\
6.39 \times 10^{-4} \\
\text { (runaway, no water- } \\
\text { loss limit) }\end{array}$ \\
\hline 2600 & 0.000501 & 0.0886 & $\begin{array}{l}0.882 \\
0.919 \\
0.955 \\
0.992 \\
1.011\end{array}$ & $\begin{array}{l}4.51 \\
4.37 \\
4.25 \\
4.13 \\
4.07\end{array}$ & $\begin{array}{c}7.71 \times 10^{-7} \\
3.13 \times 10^{-6} \\
1.18 \times 10^{-5} \\
6.94 \times 10^{-5} \\
\text { (runaway, no water- } \\
\text { loss limit) }\end{array}$ \\
\hline
\end{tabular}

Note. For a given stellar effective temperature $\left(T_{\text {eff }}\right)$, the stellar luminosity $\left(L / L_{\odot}\right)$, mass $\left(M / M_{\odot}\right)$ and incident stellar flux on the planet $\left(F_{P} / F_{\oplus}\right)$ are related to the orbital period $P$ through Equation (1). We assume synchronously rotating planets, so the orbital period $=$ rotational period. The last column is the amount of $\mathrm{H}_{2} \mathrm{O}$ available at the model top (1 mbar) and also indicates the transition stages to water loss and runaway greenhouse limits.

Figure 2 for all simulations we assume $3400 \mathrm{~K}$ blackbody stellar spectra, and we fix the planetary rotation to a 60-day synchronous orbit for all values of the incident stellar flux. The blackbody spectra and fixed 60-day rotation rate were chosen specifically to facilitate comparison with Yang et al. (2013). We also assume a completely ocean-covered world with no ocean heat transport. Here, we compare CAM simulations using our new radiative transfer code, along with several results that use the native CAM radiative transfer scheme found in versions 3 and 4 of the model (Briegleb 1992). Yang et al. (2013) used CAM3 with the spectral Eulerian dynamical core,

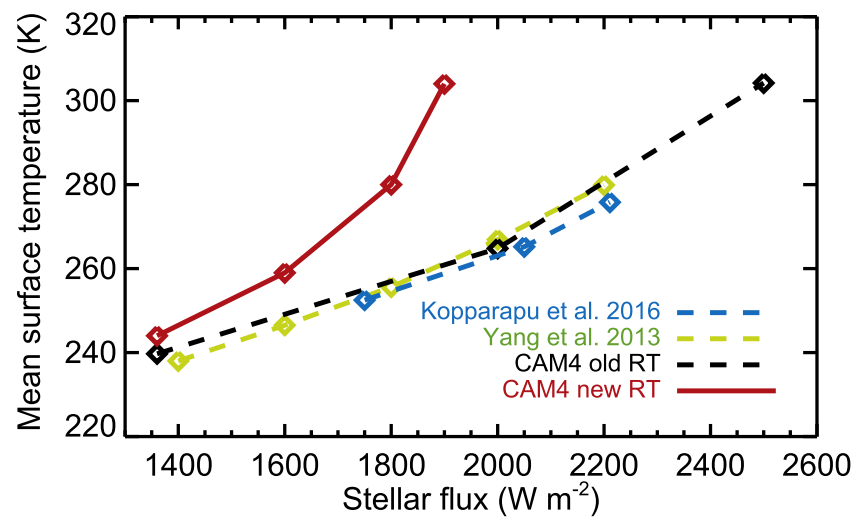

Figure 2. Comparison of CAM simulations with the native band model radiative transfer scheme (dashed) and with our correlated- $k$ radiative transfer scheme including updated $\mathrm{H}_{2} \mathrm{O}$ coefficients (red solid). Our new model indicates that a runaway greenhouse occurs at lower stellar fluxes compared with simulations that used an older version of the radiative transfer. All the simulations assumed a 60-day synchronously rotating planet in orbit around a $3400 \mathrm{~K}$ M dwarf star.

Kopparapu et al. (2016) used CAM4 with the finite-volume dynamical core, and we additionally test CAM4 with the native radiative transfer scheme and the spectral element dynamical core on a cubed-sphere grid (Lauritzen et al. 2014). Results from these three configurations, all using the native CAM radiative transfer (dashed lines), tend to cluster together irrespective of the choice in dynamical core and also independent of differences in the assumed planetary properties. Note that Yang et al. (2013) simulations assume a planet of radius $2 R_{\oplus}$ and a surface gravity of $1.4 \mathrm{~g}$, while the others assume 1 Earth mass and Earth-like gravity.

Note that some past studies have found that the CAM finitevolume dynamical core failed to capture the upper atmosphere superrotating winds of Titan and Venus (Parish et al. 2011; Lebonnois et al. 2012; Larson et al. 2014). However, here we find no resultant differences in surface climate for slow-rotating Earth-like planets using all three dynamical cores available in CAM. We are currently preparing an accompanying manuscript that focuses on the atmospheric dynamics.

Simulations with our updated radiative transfer (solid line) use the finite-volume dynamical core. These simulations yield similar temperatures under the present-day Earth stellar flux. However, as the stellar flux is increased, simulations using our updated radiative transfer code increase in temperature much more rapidly. For these control simulations, a runaway greenhouse is clearly diagnosed for stellar fluxes exceeding $1900 \mathrm{Wm}^{-2}$ using our updated code; using the native radiative transfer, however, a runaway greenhouse is not found until stellar fluxes exceed $2500 \mathrm{Wm}^{-2}$. In our new simulations, substellar clouds are still protective against high stellar fluxes, as first shown by Yang et al. (2013); however, the stronger long-wave and short-wave absorption of our model causes the the inner edge of the HZ to be shifted to lower stellar fluxes. This result comes as no surprise in light of the work of Yang et al. (2016) and the results shown here in Figure 1.

\subsection{Habitable Moist Greenhouse States}

We next conduct a suite of simulations for ocean-covered planets around late $\mathrm{K}$ and $\mathrm{M}$ dwarf stars with effective temperatures of $2600,3000,3300,3700,4000$, and $4500 \mathrm{~K}$. Note that the $2600 \mathrm{~K}$ star is roughly the same temperature as 


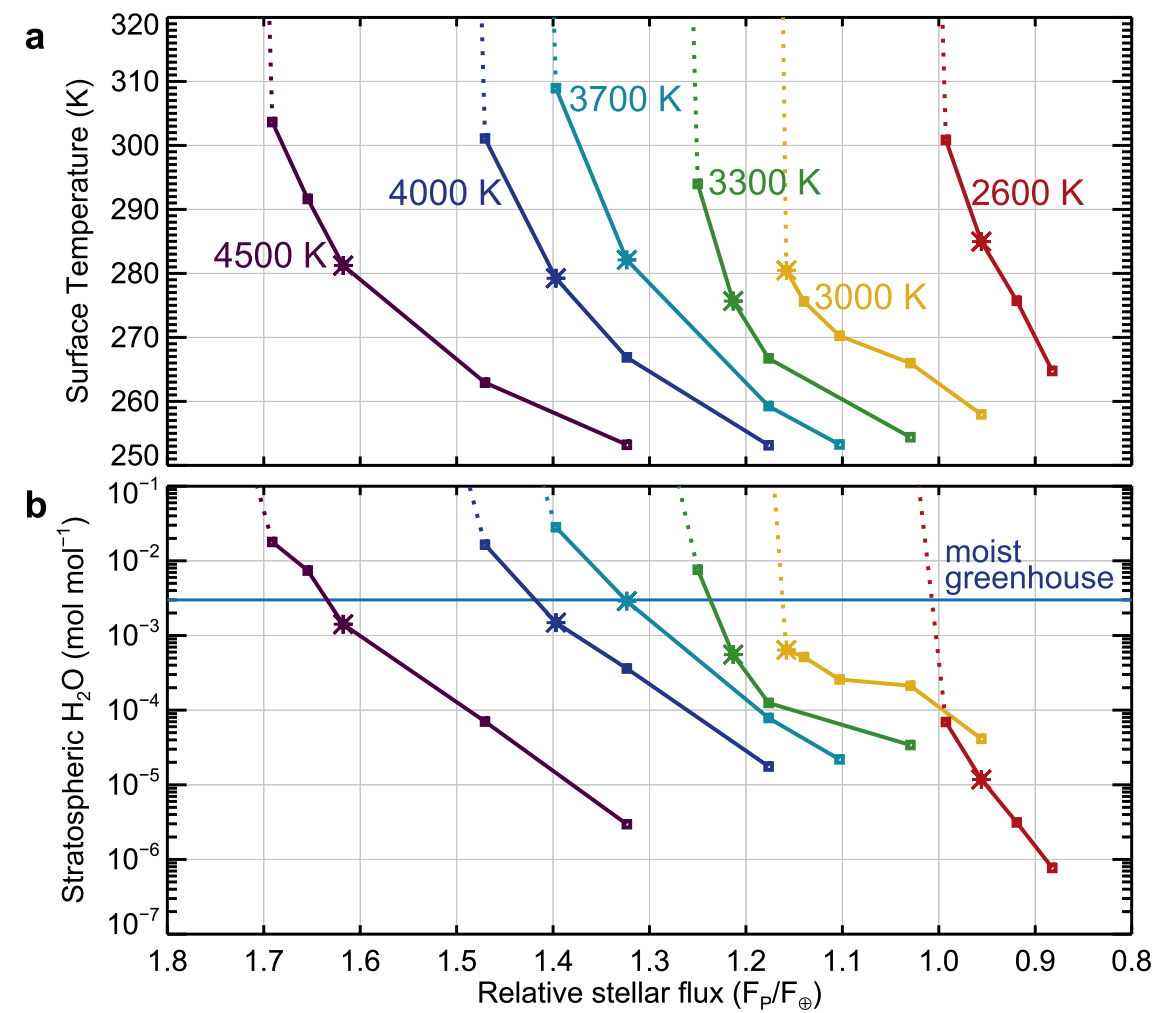

Figure 3. (a) Global mean surface temperature $\left(T_{s}\right)$ and (b) stratospheric $\mathrm{H}_{2} \mathrm{O}$ mixing ratio as a function of incident flux and stellar type. Here $F_{\oplus}=1360 \mathrm{~W} \mathrm{~m}^{-2}$. Points connected by solid curves indicate stable climate simulations, and dashed lines indicate the transition to a runaway greenhouse regime. The star symbol on the curves indicates those simulations shown in Figure 8. The consistent shift of the curves toward lower stellar fluxes from K to M dwarfs is a result of the shift in the stellar spectral distribution toward the red and shorter orbital periods that will result in inefficient substellar cloud albedo. The horizontal blue line in panel (b) indicates the $\mathrm{H}_{2} \mathrm{O}$ mixing ratio where the classical "moist greenhouse" limit occurs in 1D climate models (Kasting et al. 1993). These two panels indicate that the classical water-loss limit occurs at significantly lower surface temperatures ( 280 K) compared to the predictions of the Kasting et al. (1993) 1D model ( 340 K) for planets orbiting low-mass stars.

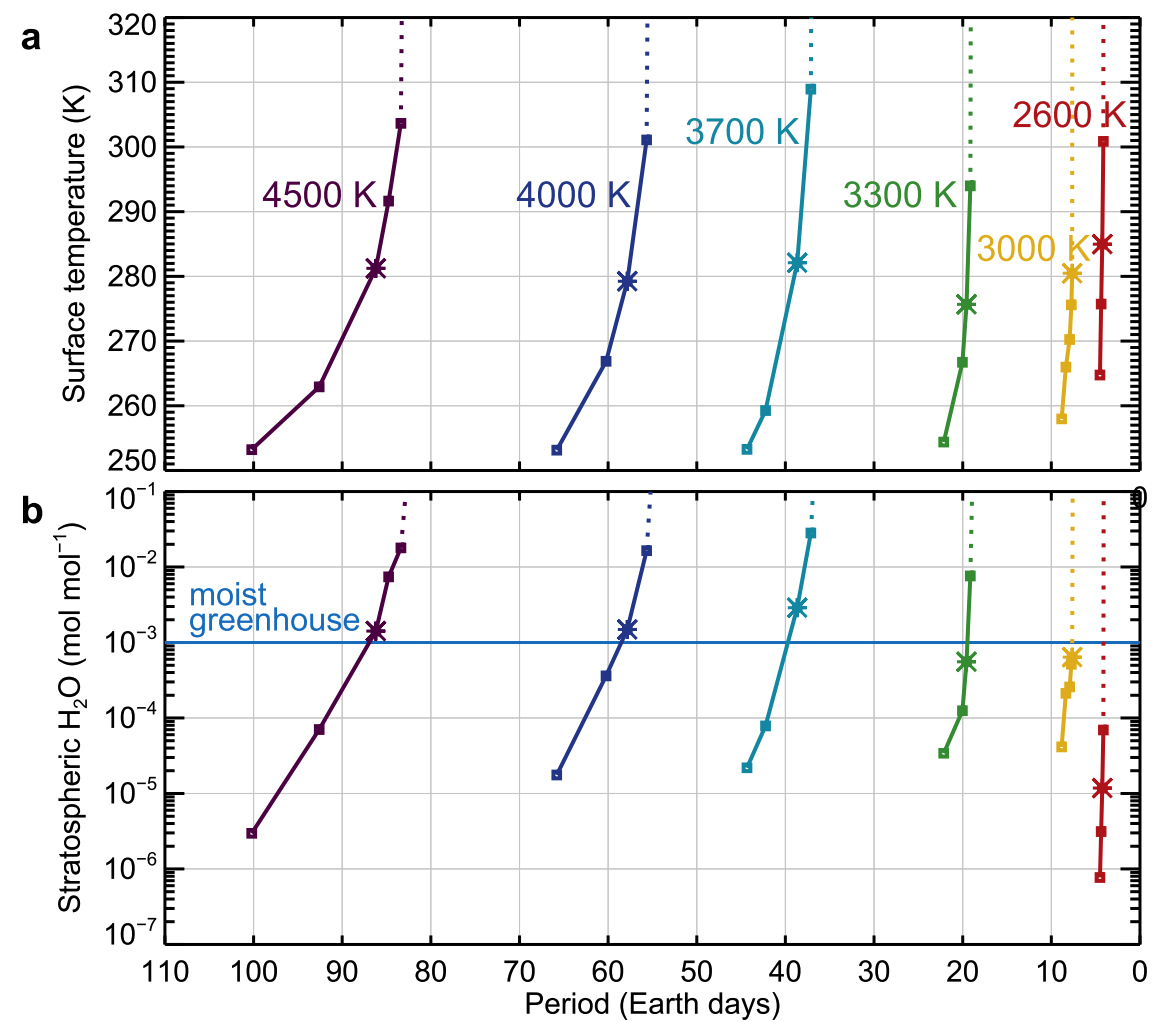

Figure 4. Similar to Figure 3, but $T_{s}$ and $\mathrm{H}_{2} \mathrm{O}$ mixing ratio as a function of orbital period of the planet. For synchronously rotating planets, this is also the rotational period. 
TRAPPIST-1, while Proxima Centauri is about $3000 \mathrm{~K}$. Our primary purpose for these numerical experiments is to determine the inner edge of the HZ for both runaway and moist greenhouse limitations. For each simulation set, we study climates with mean surface temperatures $\left(T_{s}\right)$ that range from $\sim 255 \mathrm{~K}$ up to the triggering of a runaway greenhouse, characterized by uncontrolled warming (see Section 3.3 for more details). The stellar flux and planetary rotation rate are self-consistently calculated for each case following Section 2.3. Our results are summarized in Figures 3 and 4.

Figure 3(a) shows the global mean surface temperature for each simulation versus the relative stellar flux (i.e., the ratio of the incident stellar flux on the planet to that received by Earth currently, $1360 \mathrm{~W} \mathrm{~m}^{-2}$ ). All climatologically stable simulations are shown as points connected by solid lines labeled for each given host star. Dashed lines extending upward indicate where a runaway greenhouse is triggered and climate warms uncontrollably despite only a small additional stellar forcing. Similarly, Figure 4(a) shows $T_{s}$ as a function of the orbital period of the planets. Recall that the orbital period and incident stellar flux are inextricably linked through the mass and luminosity of the host star (Kopparapu et al. 2016). Furthermore, the orbital period equals the rotational period of the planet for synchronous rotators, as are simulated here. Orbital periods for planets near the inner edge of the $\mathrm{HZ}$ are proportional to the effective temperature of the star and range from $\sim 80-100$ days around a $4500 \mathrm{~K}$ star to only $\sim 4$ days around a $2600 \mathrm{~K}$ star. Our work suggests that TRAPPSIT- $1 \mathrm{~d}$, which receives about 1.1 times current Earth flux, and whose orbital period is about 4 days, is likely in a runaway greenhouse state.

Our results also agree with Leconte et al. (2013a), who simulated synchronously rotating water-limited planets. If water is readily available on their surfaces, planet Gl 581c, at $2.5 \mathrm{~S} / \mathrm{S}_{0}$ around a $\sim 3480 \mathrm{~K}$ star, and planet HD $85512 \mathrm{~b}$, at $1.85 \mathrm{~S} / \mathrm{S}_{0}$ around a $4715 \mathrm{~K}$ star, have both likely undergone a runaway greenhouse.

The consistent shift in the simulated planet curves toward the lower incident flux as we go to cooler stars arises mainly as a result of (1) the shift in the stellar energy distribution toward the red part of the spectrum, where water vapor is a strong absorber (hence, a water-vapor rich planet around a cooler star does not need larger incident flux to quickly warm and transition to a moist or runaway greenhouse regime), and (2) the fact that the rotational periods of planets are shorter around $\mathrm{M}$ dwarf stars compared to $\mathrm{K}$ dwarfs, resulting in less efficient substellar cloud albedo, which warms the planet at lower incident fluxes.

Figures 3(b) and 4(b) show the stratospheric water-vapor volume mixing ratio as a function of relative stellar flux and orbital period, respectively. Stratospheric $\mathrm{H}_{2} \mathrm{O}$ is taken here as the value at the top of the model (1 mbar pressure). The horizontal blue line in Figures 3(b) and 4(b) indicates the classical "moist greenhouse" or water-loss limit to planetary habitability, used by Kasting et al. (1993) and Kopparapu et al. (2013). This assumes that the total planetary $\mathrm{H}_{2} \mathrm{O}$ inventory is equal to the amount of water in Earth's oceans currently: $1.4 \times 10^{24} \mathrm{~g}$ of $\mathrm{H}_{2} \mathrm{O}$. This equals $2 \times 10^{28}$ atoms $\mathrm{cm}^{-2}$. Water vapor that reaches the stratosphere is then photolyzed, releasing hydrogen, which escapes to space, thus irreversibly removing water from the planet. If one assumes that escape is diffusion limited (Hunten 1973), then the timescale for the loss of 1 Earth ocean approaches the current age of Earth when the stratospheric water-vapor volume mixing ratio is $\sim 3 \times 10^{-3}$ (Kasting et al. 1993; Kopparapu et al. 2013). This is the classical criterion of a moist greenhouse climate.

Perhaps the most interesting result evident from Figures 3 and 4 is that conditions necessary for both significant water loss and the triggering of a runaway greenhouse occur at relatively low $T_{s}$. The warmest stable climate against a runaway greenhouse is $\sim 310 \mathrm{~K}$, found for a planet around a $3700 \mathrm{~K}$ star, and it is only $\sim 280 \mathrm{~K}$ for planets around a $3000 \mathrm{~K}$ star. Furthermore, moist greenhouse conditions become evident at mean surface temperatures as low as $\sim 280 \mathrm{~K}$. Slow and synchronous rotators can have relatively large amounts of water in their atmospheres for a given $T_{s}$ owing to strong and persistent deep convection on the substellar hemisphere that continuously lofts water vapor high into the atmosphere. Thus, terrestrial planets may lose their water to space while remaining relatively cool. This is in sharp contrast to simulations of Earth around the Sun, where water-loss conditions are not typically found until $T_{s}>350 \mathrm{~K}$ (Wolf \& Toon 2015; Popp et al. 2016). Temperatures this warm, while able to support liquid surface water and extremophile life, could not support human biology (Sherwood \& Huber 2010). However, in this study, synchronously rotating planets around low-mass stars reach the moist greenhouse threshold (water-vapor mixing ratio $\sim 10^{-3}$ for 1 bar background $\mathrm{N}_{2}$ atmosphere) while maintaining robustly habitable surface conditions. A recent paper by Fuji et al. (2017) obtains a similar result using a different GCM (ROCKE-3D; Way et al. 2017), broadly consistent with our results. Note that stable moist greenhouse climates are only possible for planets orbiting host stars with $T_{\text {eff }} \geqslant 3300 \mathrm{~K}$. Planets around 3000 and $2600 \mathrm{~K}$ stars, similar to Proxima Centauri and TRAPPIST-1, bypass a water-loss phase and proceed directly to a thermal runaway, for a 1 bar background $\mathrm{N}_{2}$ atmosphere.

The low surface temperatures for the moist greenhouse regime found in this study raise unexpected and interesting prospects for planets near the inner edge of the HZ around lowmass stars. Low-mass stars may support habitable moist greenhouse worlds that could persist for several billion years during the water-loss process before becoming desiccated. Figure 5 shows the timescale to lose 1 Earth ocean of water, calculated for each simulation based on diffusion-limited escape rates. For 4500, 4000, 3700, and $3300 \mathrm{~K}$ stars, oceanloss timescales can be $>1$ Gyr for stable climates near the inner edge of the HZ. This opens the intriguing possibility that such worlds could transition from water worlds to dry planets while surface temperatures continuously remain within habitable ranges (Kodama et al. 2015). Ocean-loss timescales from stable climates around 3000 and $2600 \mathrm{~K}$ stars remain greater than 10 and $100 \mathrm{Gyr}$, respectively. Around any given star, once a runaway greenhouse instability is triggered, temperatures and thus atmospheric water vapor increase uncontrollably. Then water would be lost from the planet in only a few million years, and the surface would be sterilized.

\subsection{Transition to Runaway Greenhouse}

In our simulations we can definitively identify the triggering of a runaway greenhouse. Figure 6 shows time series of temperature, planetary albedo, and the top-of-atmosphere (TOA) energy balance for several stable simulations and for one simulation that enters a runaway greenhouse. While 


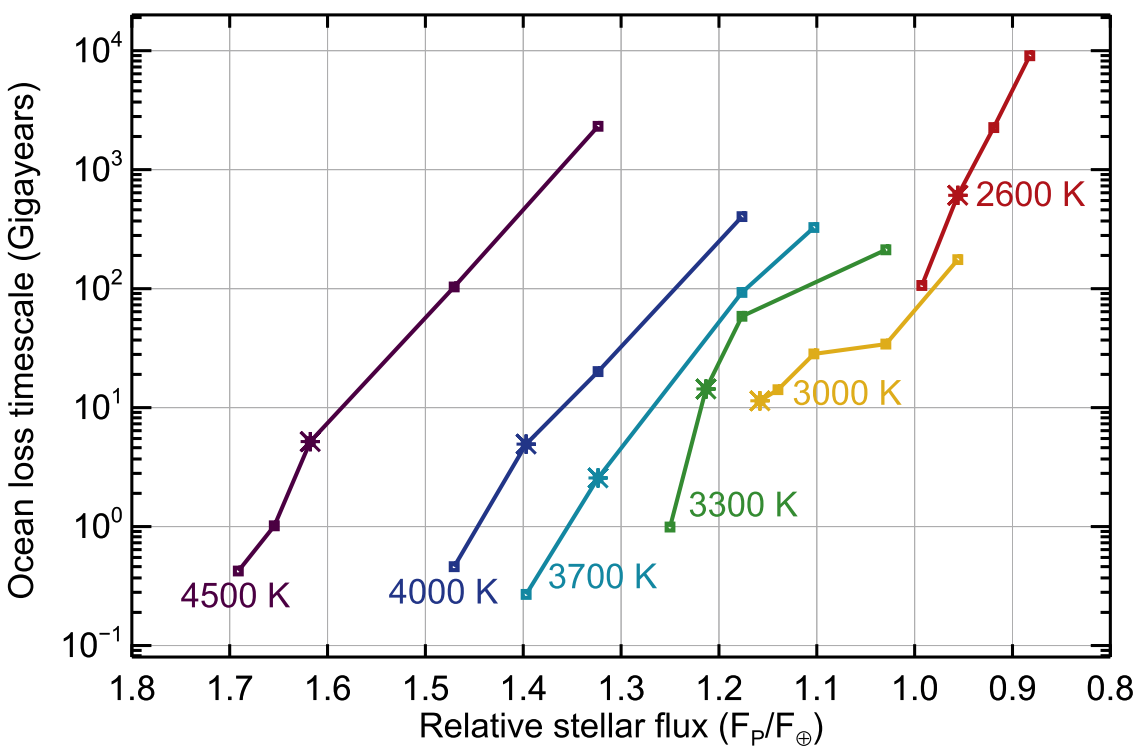

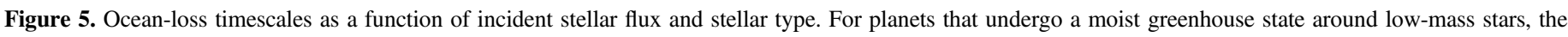
ocean-loss timescales can be $>1$ Gyr while maintaining lower surface temperatures $(\sim 280 \mathrm{~K})$.

numerical limitations of the model prevent us from exploring the end state of the runway process where $T_{s} \sim 1600 \mathrm{~K}$ (Goldblatt et al. 2013), still we can mark where the energy imbalance grows large and temperatures begin to rise uncontrollably. This is sufficient for constraining the inner edge of the HZ for these worlds. Figure 6 shows time series model outputs only for simulations around a $3700 \mathrm{~K}$ star; however, a similar temporal evolution is found for each stellar type studied here. In this case, climatologically stable simulations maintain a planetary albedo of $\sim 0.45$, and the TOA energy balance naturally approaches zero. The runaway simulation experiences a collapse of the planetary albedo (dropping to $\sim 0.1$ ), coincident with a ballooning of the TOA energy balance. At the time of model termination, the global mean surface temperature exceeds $350 \mathrm{~K}$, the maximum atmosphere temperature exceeds $400 \mathrm{~K}$, and the TOA energy imbalance has risen to nearly $+150 \mathrm{Wm}^{-2}$.

For synchronously rotating planets around low-mass stars, the triggering of a runaway greenhouse is intimately linked to the thick substellar cloud deck. Figure 7 illustrates the time evolution of the vertical temperature structure and vertical cloud fraction of the atmosphere at the substellar point during the beginning stages of an ensuing runaway greenhouse. The results in Figure 7 correspond to the runaway greenhouse case also shown in Figure 6.

In the opening (Earth) years of simulation, the runaway case maintains thick clouds at the substellar point (Figure 7(b)) while the planetary albedo remains large (Figure 6(b)), similar to the stable cases. However, as temperatures continue to warm, a radiative-convective transition occurs in the low atmosphere. As described by Wolf \& Toon (2015), increasing water vapor in the atmosphere for warming climates causes the low atmosphere to become opaque to thermal radiation, while absorption of incident stellar radiation increases. In total, this causes net radiative heating of the low atmosphere, which forms a strong temperature inversion (Figure 7(a)). The inversion encompasses the entire planet, including the substellar point, and stabilizes the low atmosphere against convection. Inversion layers (such as Earth's stratosphere) are inherently stable against vertical mixing. Without deep convection carrying moisture up from the boundary layer, the once thick and highly reflective, convectively produced substellar cloud deck dissipates completely. High temperatures within the inversion layer led to low relative humidities and clear skies, despite a considerable water-vapor burden. In the absence of these substellar clouds, the planetary albedo plummets and the planet is left unprotected against large stellar fluxes. The energy imbalance skyrockets, and a thermal runaway becomes inevitable.

\subsection{Dependence on the Host Star}

The climates of planets in the HZs of low-mass stars are inextricably controlled by the nature of the host star. The orbital period, total incident stellar flux, and stellar energy distribution are all correlated, determined by the mass and luminosity of the host star. Higher-mass stars are bluer and brighter, and thus planets in their $\mathrm{HZ}$ must reside at further distances and thus will have longer periods and slower rotation rates. Here we find that planets near the inner edge of the $\mathrm{HZ}$ around $4500 \mathrm{~K}$ stars will have periods of $\sim 80-100$ Earth days (Figure 4). Lower-mass stars are redder and dimmer; thus, HZ planets must remain closer to their host star and on shorter periods. For instance, near the inner edge of the HZ planets around a $2600 \mathrm{~K}$ star have orbital periods of only $\sim 4-5$ days (Figure 4). Differences in the rotation rate of these planets are of critical importance, because the rotation rate of the planet imposes a strong control on the atmospheric circulation regime through the modulation of the Coriolis force. Slow rotation weakens the Coriolis effect and causes the atmospheric circulation to shift from a "rapidly rotating" regime characterized by zonal uniformity and symmetry about the equator (e.g., like Earth currently) to a "slow-rotating" regime characterized by dayside to nightside transport and circular symmetry about the substellar point. The transition between these circulation regimes occurs when the rotation rate exceeds $\sim 5$ days for a $1 R_{\oplus}$ planet (Yang et al. 2014). Thus, planets near the inner edge of the $\mathrm{HZ}$ around a $2600 \mathrm{~K}$ star with a $\sim 4$-day rotation rate (like TRAPPIST-1d) should be in the fast-rotating circulation regime. Planets near the inner edge of the HZ 


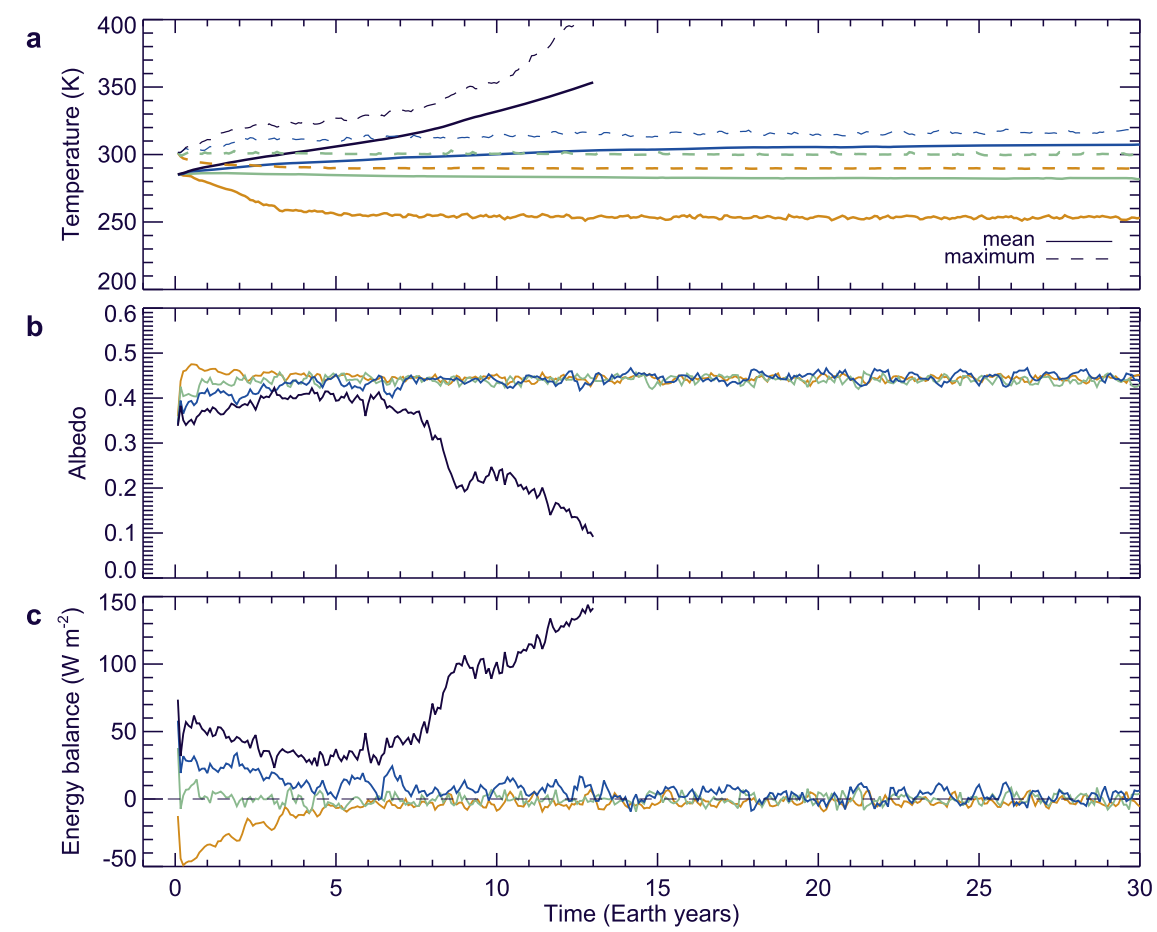

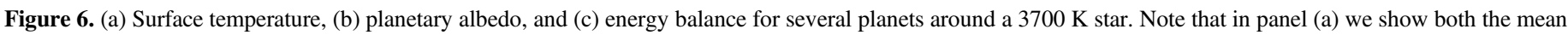

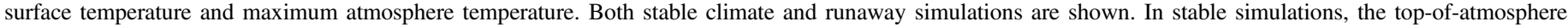

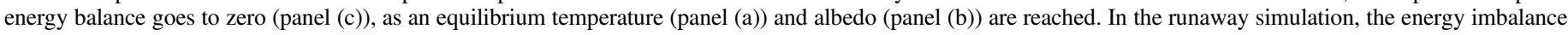
becomes large and temperatures begin to increase uncontrollably.

around $3000 \mathrm{~K}$ star have rotations rates of $\sim 8$ days and thus may represent a transitional state between rapid and slow rotators. All other cases studied lie firmly within the slowrotating regime. The circulation regime critically feeds back on the planets' distribution of clouds.

Figure 8 shows contour plots of surface temperature, TOA albedo, integrated cloud water column, and integrated cloud fraction for a present-day Earth control simulation, and then for $\mathrm{HZ}$ planets around each of our six low-mass stars studied here. Note that the incident stellar flux on the planet, the planet's rotational period, and the planet's mean surface temperature are listed at the left margin of Figure 8. The white line overplotted on the surface temperature contour indicates the location of the sea-ice margin. The substellar point is located at the center of each panel. All simulations shown in Figure 8 have moderate surface temperatures, with $276 \mathrm{~K} \leqslant T_{s} \leqslant 288 \mathrm{~K}$. We have chosen to display simulations with similar $T_{s}$, because the mean temperature of the planet dictates sea ice and the atmospheric water vapor, both of which impose primary controls on planetary climate. By comparing simulations of nearly the same temperature (despite varying stellar flux, period, and spectra), we can normalize against temperature-dependent feedbacks on climate.

The climates shown in Figure 8 are modulated by the rotation rate of each planet, through the feedback between the large-scale circulation and water clouds. The TOA albedo is largely controlled by the fraction and thickness of the clouds. Note that in Figure 8 dark (light) colors represent areas of low (high) albedo for the planets in the second column. The patterns emergent in planetary albedo mirror the distribution of the cloud water column and cloud fraction. Regions of thick and ubiquitous clouds are naturally coincident with regions of high albedo. Fast rotators, like present-day Earth and planets near the inner edge of the $\mathrm{HZ}$ around ultra-low-mass stars (i.e., $2600 \mathrm{~K}$ in this study), exhibit zonal patterns of temperature and clouds. These climates feature the fewest clouds and the lowest albedos and thus reach Earth-like temperatures at relatively low values of the incident stellar flux. For planets around a $2600 \mathrm{~K}$ star, while the overall pattern is zonal, the planet retains the signature of a substellar hot spot owing to its synchronous rotation. Around increasingly higher $T_{\text {eff }}$ stars, the rotational period of $\mathrm{HZ}$ planets becomes necessarily longer and the circulation regime shifts. Substellar clouds become increasingly thick and ubiquitous, resulting in higher planetary albedos and larger required incident stellar fluxes to maintain Earth-like temperatures.

\subsection{Atmospheric Circulation}

The zonal circulation is the predominant circulation pattern on synchronously rotating planets, providing the primary means of energy transport from the substellar to antistellar hemisphere. Figure 9 shows the mean zonal circulation (MZC, also known as the Walker circulation) for planets in synchronous rotation around stars with $T_{\text {eff }}=3300 \mathrm{~K}$ and $T_{\text {eff }}=2600 \mathrm{~K}$ (top row), as well as in units of pressure tendency. Note that pressure tendency is a commonly used unit for expressing vertical motion in the atmosphere, i.e., a negative pressure tendency refers to rising motion, and vice versa.

We note that a planet in a stable 4.25-day orbit near the inner edge of the HZ around a star with $T_{\text {eff }}=2600 \mathrm{~K}$ rotates fast enough that the Rossby deformation radius is less than the planetary radius. Thus, while the planet around the $T_{\text {eff }}=3300 \mathrm{~K}$ star is able to sustain strong rising motion at the substellar point and a hemisphere-spanning MZC from the dayside to nightside, the planet around the $T_{\text {eff }}=2600 \mathrm{~K}$ star 

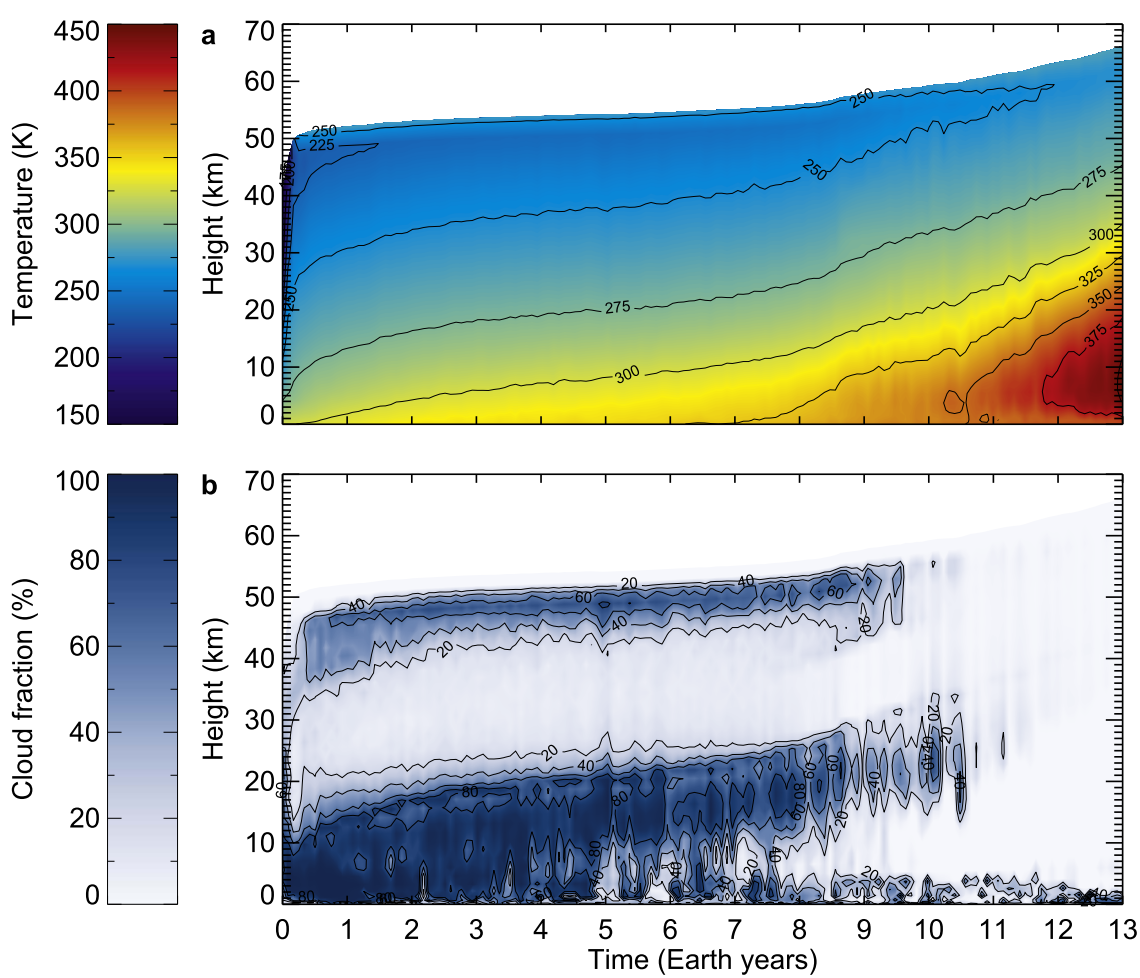

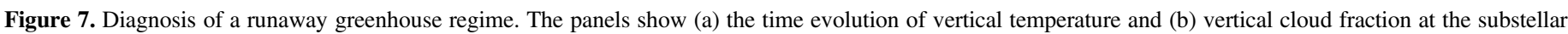

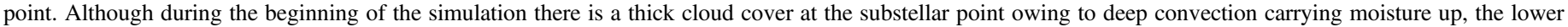

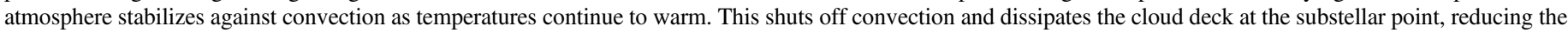
planetary albedo, and energy imbalance ensues owing to large incident stellar flux.

lacks a region of strong convection beneath its substellar point and shows a less organized structure than the time-average MZC. Strong convection is also absent in the time-average MZC for the $24 \mathrm{hr}$ rotating planets, where the lack of a fixed substellar point indicates the presence of complex, diurnally varying MZC patterns closer to those observed on Earth today. Differences between the planet orbiting the Sun and Ad Leo are small, with planetary rotation rate being the prevailing factor for predicting differences in atmospheric circulation.

\subsection{Inner Edge of the Habitable Zone}

Figure 10(a) shows our simulated model cases around stars with $2600 \mathrm{~K} \leqslant T_{\text {eff }} \leqslant 4500 \mathrm{~K}$. The blue-square simulations have mild climates, with low stratospheric $\mathrm{H}_{2} \mathrm{O}$, whereas the green squares have TOA water vapor of $10^{-3}$ or greater ("moist stratosphere"). Red crosses are simulations where the planet is in thermal runaway. For planets in synchronous orbits around stars with $T_{\text {eff }}>3000 \mathrm{~K}$, there is an intermediate stage where the upper atmosphere is dominated by water vapor, before the planet transitions to a runaway with an increase in stellar flux. For stars with $T_{\text {eff }} \leqslant 3000 \mathrm{~K}$, the planet directly transitions to a runaway from a stable mild climate. As discussed in previous sections, this abrupt transition to a thermal runaway, without undergoing a water-vapor-dominated atmosphere, arises partly as a result of the shorter orbital periods (and hence faster rotation rates), which advect the persistent substellar cloud deck to the nightside, decreasing the planetary albedo and increasing the surface temperatures. This effect is compounded by the shift in the peak wavelength of the stellar spectra toward the near-IR for low-mass stars, where $\mathrm{H}_{2} \mathrm{O}$ vapor is a strong absorber. Our updated absorption coefficients for water vapor further magnify this effect, leading to the runaway greenhouse phase for any further increase in the stellar flux.

Figure 10(b) shows our moist and runaway greenhouse limits in comparison with our previous study (Kopparapu et al. 2016) and Yang et al. (2014a). Note that, as with Yang et al. (2014a), Kopparapu et al. (2016) also assumed that the last converged solution represents the IHZ. ${ }^{10}$ We assumed a solar metallicity for all the stars in this study. Compared to our previous results, the inner edge of the $\mathrm{HZ}$ (either moist or runaway $\mathrm{GH}$ ) is located at considerably lower stellar fluxes for late $\mathrm{K}$ and $\mathrm{M}$ dwarf stars. Considering that the global mean surface temperatures at the moist greenhouse limit are $\sim 280 \mathrm{~K}$, we conclude that, for $\mathrm{M}$ dwarf stars, the inner edge of the $\mathrm{HZ}$ is identified by the runaway greenhouse limit and moist greenhouse planets around these stars can be habitable, revising decades of previous assumptions about the moist greenhouse.

We remind the reader that our simulations assume a background $\mathrm{N}_{2}$ atmosphere of 1 bar. Several studies have shown that the variation in the noncondensable gas fraction in the atmosphere (specifically the $\mathrm{N}_{2}$ amount) can affect the OLR and consequently the habitability limits through moist and runaway greenhouse (Nakajima et al. 1992; Pierrehumbert 2010; Kopparapu et al. 2014; Wordsworth \& Pierrehumbert 2014). For a higher $\mathrm{N}_{2}$ amount in the atmosphere, the surface temperature needs to be higher to radiate the same amount of OLR. The reason for this is that the amount of water-vapor fraction in the atmosphere is not high enough relative to the $\mathrm{N}_{2}$ fraction. Consequently, planets with higher $\mathrm{N}_{2}$ can potentially reach moist greenhouse limits at higher temperatures than $\sim 280 \mathrm{~K}$. Conversely, if there is less than 1 bar $\mathrm{N}_{2}$ in the

\footnotetext{
${ }^{10}$ For a detailed analysis of the last converged solution criterion, see Section 3.4 of Kopparapu et al. (2016).
} 


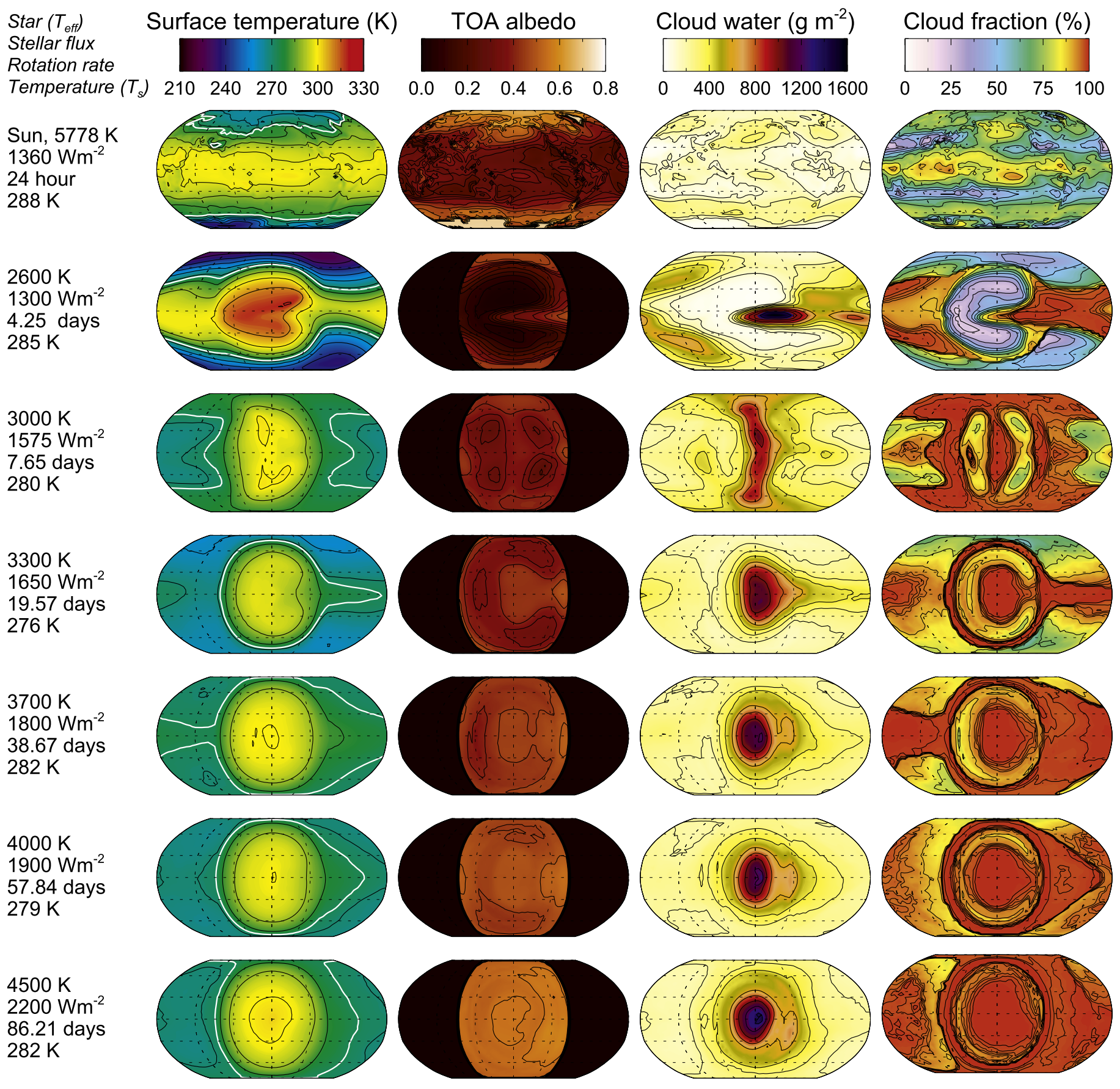

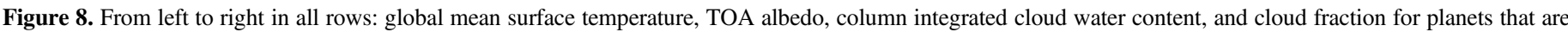

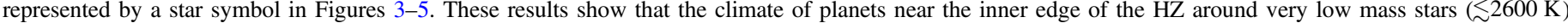
resembles the climate of fast-rotating planets like Earth.

atmosphere, then water loss could occur at even lower temperatures. Still, our assumption of a 1 bar $\mathrm{N}_{2}$ atmosphere is in line with previous studies (Kasting et al. 1993; Kopparapu et al. 2013; Leconte et al. 2013b; Yang et al. 2014; Wolf \& Toon 2015; Popp et al. 2016). An exploration of the effects of varying $\mathrm{N}_{2}$ on moist greenhouse atmospheres in $3 \mathrm{D}$ models is left for future parameter studies.

We further remind the reader that differences exists across $3 \mathrm{D}$ climate models of varying origin. For example, outcomes for standard anthropogenic climate change scenarios can vary by several kelvin over the next two centuries, depending on the model being used (Rogelj et al. 2012). However, the implied radiative forcings for simulated exoplanets are typically much larger and yield wider spreads in model results (e.g., Popp et al. 2016; Figure 7). Formal model intercomparisons are needed in order to make sense of these model differences.

\section{Discussion}

\subsection{Transit Transmission Spectral Features}

M dwarf planets will be targeted by JWST, and if habitable planets can exist closer to these stars than the traditional HZ boundaries, their shorter orbital periods will allow us to observe them more frequently. For example, the TRAPPIST-1 

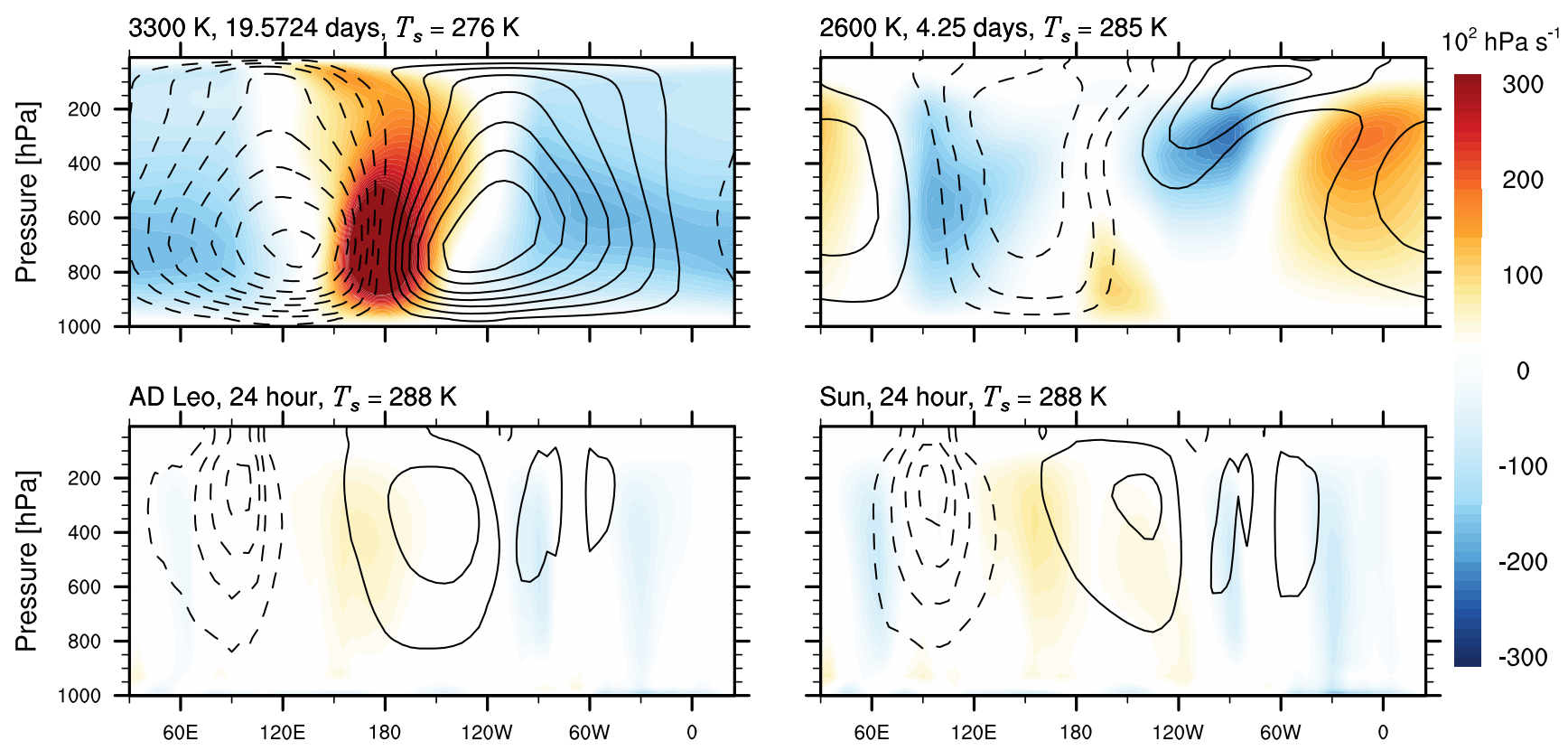

Figure 9. Mean zonal circulation (MZC) and vertical wind for synchronously rotating planets around 3300 and $2600 \mathrm{~K}$ stars (top) and rapidly rotating $24 \mathrm{hr}$ period planets around AD Leo and the Sun (bottom). The contour interval for the MZC is $30 \times 1011 \mathrm{~kg} \mathrm{~s}^{-1}$, with solid contours indicating clockwise circulation and dashed contours indicating counterclockwise circulation. Shading indicates pressure tendency, which corresponds to rising (warm colors) or sinking (cool colors) motion.

system has three planets (d, e, and f) in the habitable zone. TRAPPIST-1 is visible for a total of 99 days in JWST's Cycle 1 (STScI APT). Planets d, e, and $\mathrm{f}$ have orbital periods of 4,6 , and 9 days, respectively. Therefore, in cycle 1 (1 yr), planet $\mathrm{f}$ can be observed for a maximum of 11 transit observations, whereas planet $d$ can be observed for a maximum of 24 transits. TRAPPIST-1 has a stellar effective temperature of $2600 \mathrm{~K}$, so it is not directly applicable to the moist greenhouse atmospheres discussed here. However, it does offer an estimate for what a feasible amount of observing time is for JWST. Therefore, if we use TRAPPIST-1's observability window as a standard estimate, we can calculate the expected noise on the transmission spectra of the planets of interest in this study (moist greenhouse planets around stars with $T_{\text {eff }}>3300 \mathrm{~K}$ ). In Figure 11, we show a simulated transit transmission spectrum from our GCM simulations, where the planet is undergoing moist greenhouse around an M3 spectral type star with $T_{\text {eff }}=3300 \mathrm{~K}$ and stellar radius of $0.137 R_{\oplus}$ (dark blue). All spectra were generated using the SMART model, a versatile line-by-line 1D radiative transfer model (Meadows \& Crisp 1996). Transit spectra are generated from GCM results by using atmospheric columns from the planetary terminator region. This planet's atmosphere includes $\mathrm{N}_{2}$ as the background gas with stratospheric $\mathrm{H}_{2} \mathrm{O}$ volume mixing ratio of $10^{-3}$ (see Figures 3 and 4) and self-consistent liquid and ice water clouds. Figure 11 shows that for a habitable moist greenhouse planet, there are two prominent water-vapor features, including one between 5 and $8 \mu \mathrm{m}$, within the JWST MIRI LRS (Mid InfraRed Instrument Low Resolution Spectrometer) bandpass, and another between 2.5 and $3 \mu \mathrm{m}$, within the NIRCam grism with the F322W2 filter and the NIRSpec G235M/H bandpasses. These features, caused by a wet upper atmosphere, are a direct consequence of the strong and persistent convection that occurs at the substellar region for slowly rotating planets. This strong substellar convection is effective at pumping water vapor into the high atmosphere.
In Figure 11, we also show a planet simulated around an $\mathrm{M}$ dwarf, but with a standard $24 \mathrm{hr}$ rotation period (light-blue spectrum). Previous studies of Earth-analog spectra for planets in the $\mathrm{HZ}$ around $\mathrm{M}$ dwarfs have not included the impact of synchronous rotation (Barstow et al. 2015; Schwieterman et al. 2016). Taking into account the effect of $M$ dwarf rotation on cloud and species distributions will be crucial to accurately model the spectra of these worlds. Note how the planet simulated with a $24 \mathrm{hr}$ rotation period results in a dramatically different spectrum in Figure 11 compared to including synchronous rotation. The water-vapor spectral feature is $\sim 7$ times weaker in the atmosphere of the planet with the $24 \mathrm{hr}$ rotation period because the strong substellar convection is absent.

Despite the increased strength of the water absorption with the inclusion of synchronous rotation, the two strongest water spectral features in Figure 11 are well below the postulated JWST noise floor of 20 and $50 \mathrm{ppm}$ for the near-IR and mid-IR instruments aboard JWST (Greene et al. 2016). In reality, though, noise floors will not prevent observations of potentially habitable systems, and the true noise floor of JWST remains to be determined. For example, TRAPPIST-1 is a compelling enough target that it is already on JWST's Guaranteed Time Observer's plan. If other compelling small planets are found (planets that may fit the planet type described here), they may end up being observed, and so we should address what, if anything, we could learn from their transmission spectra.

Assuming that JWST encounters no systematic noise floor (which is unlikely), we can calculate how many observing hours would be needed to obtain sub-10 ppm precision. Noise floors are created when instrument systematics cannot be removed from co-adding transits. Therefore, they create a minimum level equal to the strength of the systematic effect. Figure 12 shows the approximate error on the spectrum of an MIRI observation at $6 \mu \mathrm{m}$ for a range of stellar brightnesses (2MASS J), without any inclusion of a systematic noise floor. We chose the MIRI band because of the broadness of the $6 \mu \mathrm{m}$ 

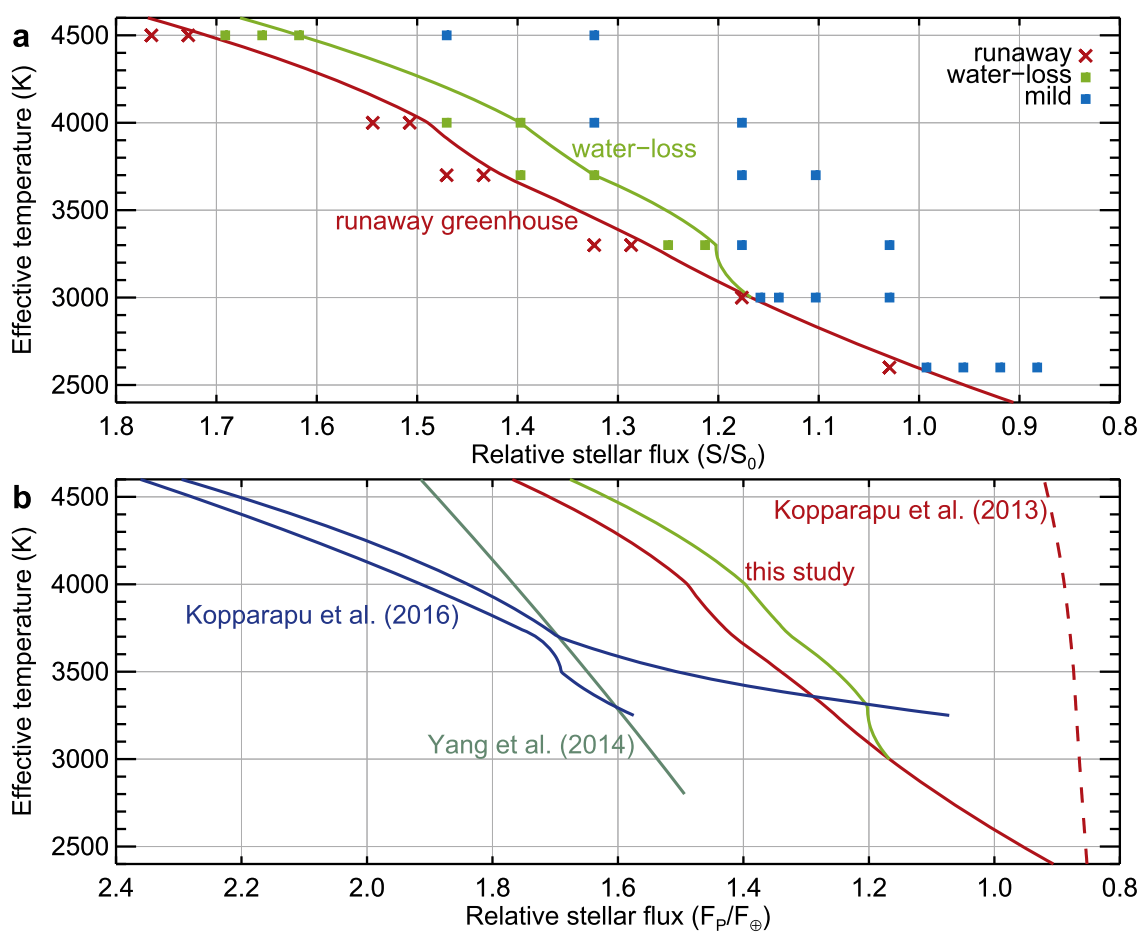

Figure 10. Results of aquaplanet model simulations, after updating our GCM with new $\mathrm{H}_{2} \mathrm{O}$ absorption coefficients. (a) For planets around stars with $T_{\text {eff }} \geqslant 3000 \mathrm{~K}$, there is an intermediate stage between mild climates (blue squares) and thermal runaway (red crosses), where the upper atmosphere is dominated by water vapor (green squares) that could result in water loss. Planets with $T_{\text {eff }}<=3000 \mathrm{~K}$ directly transition to runaway with an increase in stellar flux due to faster rotation rates, which advect the persistent substellar cloud deck to the nightside, decreasing the planetary albedo and increasing the surface temperatures. (b) Comparison of our new inner $\mathrm{HZ}$ results with previous studies.

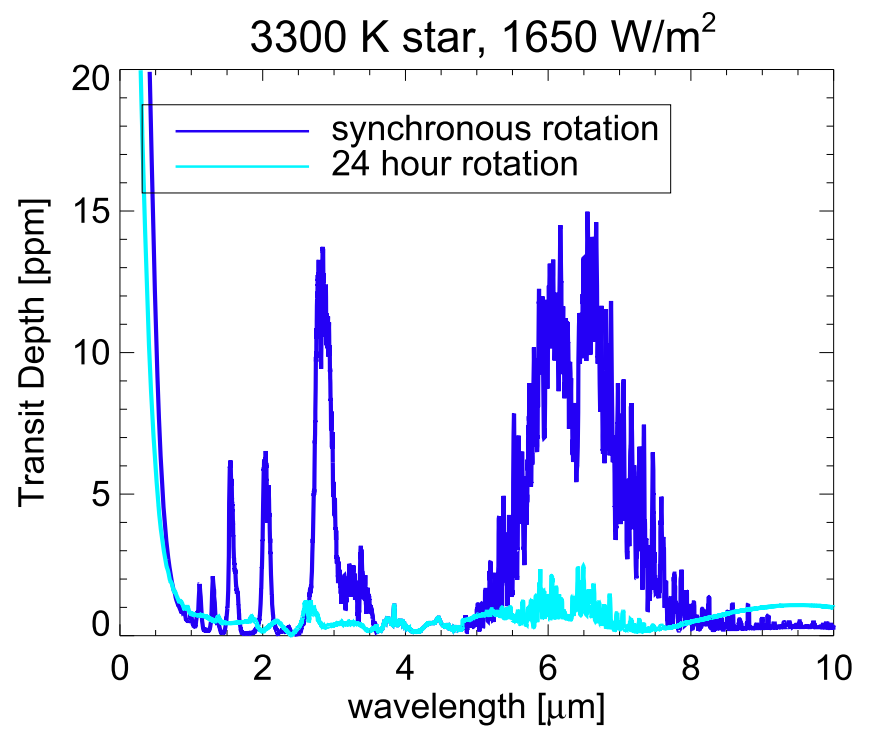

Figure 11. Water-vapor features are prominent in the moist greenhouse atmosphere of an Earth-sized planet orbiting a $3300 \mathrm{~K}$ star, with a global mean surface temperature of $\sim 285 \mathrm{~K}$, when synchronous rotation is accounted for using GCMs (dark blue). Using an Earth-analog planet with $24 \mathrm{hr}$ rotation (light blue) around a $\mathrm{M}$ dwarf will miss these water-vapor features in the spectra.

water feature and because, as we are not including any systematic noise floor and observing an M3 star, the noise calculations are similar if the near-IR simulations are binned to $R=100$. These calculations were performed using JWST's

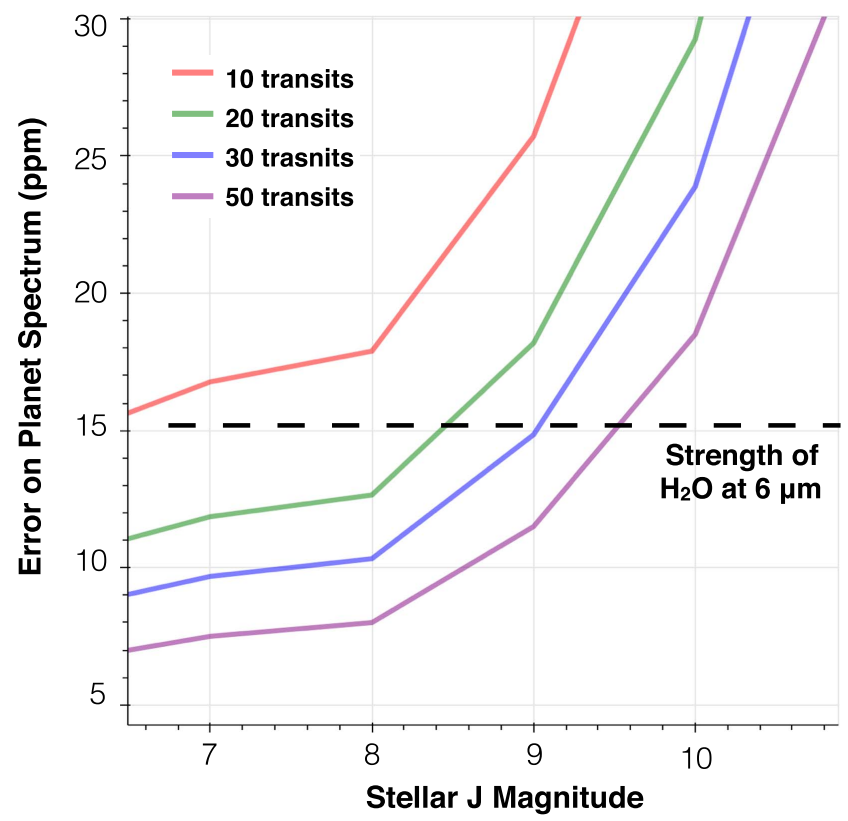

Figure 12. Noise estimates calculated with PandExo at $6 \mu \mathrm{m}$ with JWST's MIRI instrument. The system simulated was an M3 stellar spectral type with $T=3300 \mathrm{~K}$. Each transit consists of a $2 \mathrm{hr}$ in-transit observation with a $2 \mathrm{hr}$ out-of-transit baseline. At $J=6$, the duty cycle is calculated to be $33 \%$ with two groups/integration. As the target gets dimmer, the duty cycle increases to $98 \%$ at $J=11$ with 130 groups/integration. No systematic noise floor has been added, although it is postulated to be $\sim 50 \mathrm{ppm}$ (Greene et al. 2016), based on comparisons with Spitzer and not measurements from the JWST MIRI team. The dashed line signifies the strength of the water absorption feature for the synchronously rotating system in Figure 11. 
publicly available noise simulator, PandExo (Batalha et al. 2017), for the same planet system shown in Figure 11. Assuming an observability window comparable to TRAPPIST-1 and an inner edge planet, the planet system would need to be observed through the entirety of Cycle 1 (24 transits) to reach the approximate strength of the water feature.

Solely surpassing the strength of the water feature would yield a confirmation of the presence of water, not necessarily a constraint on the water abundance. In this particular scenario (aquaplanet, no land), if the presence of water is confirmed, one could say that the planet is in either a moist greenhouse or a habitable state given its stellar type and orbital period (see Figure 4). If the system was then observed through Cycles $\sim 2-3$ ( $\sim 60$ transits), a traditional Bayesian retrieval might be possible to constrain the water mixing ratio and corresponding temperature (such as that done in Barstow et al. 2016). Barstow et al. (2016) estimate retrieved errors on water abundance on the order of \pm 1 dex in mixing ratio. If it is possible to measure the water content to a level of precision of approximately \pm 1 dex, there would be three potential scenarios in accordance with Figure 4 (habitable, entering moist greenhouse, in moist greenhouse). Since transmission spectra will not probe the surface of terrestrial planets, we can use Figure 4 to predict a surface temperature. This would require constraints on the stratospheric temperature, the water mixing ratio, and information on the stellar type and orbital period.

In a scenario where by the end of Cycle 3 there is still no detection of any water spectral features, two possibilities exist: either the planet is a fast rotator so that strong substellar convection is absent, or the planet is dry. Both scenarios would be astrophysically interesting. Of course, there are other factors, which may complicate this simple qualitative view of exoplanet characterization. For example, here we have assumed an aquaplanet with no land. The effect of adding continents may also result in reduced $\mathrm{H}_{2} \mathrm{O}$ features. An exploration of these dependencies is left as future work.

\section{Conclusions}

We have updated water-vapor absorption coefficients in our CAM 3D GCM with the HITRAN 2012 LBL database and added continuum absorption. Our results indicate, assuming synchronously rotating Earth-sized and Earth-massed planets with background 1 bar $\mathrm{N}_{2}$ atmospheres, that the inner edge of the $\mathrm{HZ}$ for late $\mathrm{M}$ to mid-K dwarfs is farther away from the star than previous studies. Furthermore, for stars with $T_{\text {eff }} \geqslant 3300 \mathrm{~K}$, there appears to be a habitable moist greenhouse regime, which could not be predicted with $1 \mathrm{D}$ climate models. For stellar $T_{\text {eff }} \leqslant 3000 \mathrm{~K}$, planets at the inner $\mathrm{HZ}$ directly transition to runaway phase. We find that the transition between synchronous and rapidly rotating planets occurs at an orbital period of $\sim 4$ days, where the substellar cloud advected to the nightside, decreasing the planetary albedo and increasing the surface temperatures. We also find that using Earth-analog spectra for planets orbiting $M$ dwarfs underestimates the strength of the critical water-vapor feature because the $24 \mathrm{hr}$ rotation period of Earth does not produce strong substellar convection that transports water to the upper atmosphere. This strong convection is a feature of synchronously rotating planets as pointed out by several GCM studies, including this paper. Therefore, to interpret the spectral features of $\mathrm{HZ}$ planets around $\mathrm{M}$ dwarf stars, one needs to employ 3D climate models to properly account for the effect of rotation on the atmosphere of the planet.

The authors would like to thank David Paynter for providing BPS coefficients. We also thank an anonymous reviewer for helpful comments. R.K., E.T.W., and J.H.-M. gratefully acknowledge funding from NASA Habitable Worlds grant NNX16AB61G. R.K. and G.A. also acknowledge funding from NASA Astrobiology Institute's Virtual Planetary Laboratory lead team, supported by NASA under cooperative agreement NNH05ZDA001C. N.E.B. acknowledges support from the National Science Foundation under grant no. DGE1255832. The Center for Exoplanets and Habitable Worlds is supported by the Pennsylvania State University, the Eberly College of Science, and the Pennsylvania Space Grant Consortium. This work was facilitated through the use of advanced computational, storage, and networking infrastructure provided by the Hyak supercomputer system, supported in part by the University of Washington eScience Institute. This work also utilized the Janus supercomputer, which is supported by the National Science Foundation (award no. CNS-0821794) and the University of Colorado at Boulder. Any opinions, findings, and conclusions or recommendations expressed in this material are those of the author(s) and do not necessarily reflect the views of NASA or the National Science Foundation.

\section{ORCID}

Eric T. Wolf (iD https://orcid.org/0000-0002-7188-1648 Giada Arney (D) https://orcid.org/0000-0001-6285-267X Natasha E. Batalha (i) https://orcid.org/0000-0003-1240-6844 Jacob Haqq-Misra (ib https://orcid.org/0000-0003-4346-2611

Simon L. Grimm (ib https://orcid.org/0000-0002-0632-4407 Kevin Heng (ib https://orcid.org/0000-0003-1907-5910

\section{References}

Abe, Y., Abe-Ouchi, A., Sleep, N. H., et al. 2011, AsBio, 11, 443 Airapetian, V. S., Glocer, A., \& Khazanov, G. V. 2017, ApJL, 836, 3 Allard, F., Allard, N. F., Homeier, D., et al. 2007, A\&A, 474, L21

Allard, F., Guillot, T., Ludwig, H. G., et al. 2003, in Proc. IAU Symp. 211, Brown Dwarfs, ed. E. Martin (San Francisco, CA: ASP), 325 Anglada-Escudé, G., Amado, P. J., Barnes, J., et al. 2016, Natur, 536, 437 Arney, G., Domagal-Goldman, S. D., Meadows, V. S., et al. 2016, AsBio, 16,837

Arney, G., Meadows, V. S., \& Crisp, D. 2014, JGR, 119, 1860

Baraffe, I., Chabrier, G., Allard, F., \& Hauschildt, P. H. 2002, A\&A, 382, 563 Barker, H. W., Cole, J. N. S., Morcrette, J. J., et al. 2008, QJRMS, 134, 1463 Barnes, R., Deitrick, R., Luger, R., et al. 2016, arXiv:1608.06919

Barnes, R., Mullins, K., \& Goldblatt, C. 2013, AsBio, 13, 225

Barstow, J. K., Aigrain, S., Irwin, P. G., et al. 2015, MNRAS, 458, 2657 Barstow, J. K., Aigrain, S., Irwin, P. G. J., et al. 2016, MNRAS, 458, 3 Batalha, N. E., Mandell, A., Pontoppidan, K., et al. 2017, PASP, 129, 064501 Bitz, C. M., Shell, K. M., Gent, P. R., et al. 2012, JCli, 25, 3053 Bolmont, E., Selsis, F., \& Owen, J. E. 2017, MNRAS, 464, 3728 Boyajian, T. S., Von Braun, K., van Belle, G., et al. 2013, ApJ, 771, 40 Briegleb, B. P. 1992, JGR, 97, 7603

Brent, R. P. 1973, Algorithms for Minimization without Derivatives (Englewood Cliffs, NJ: Prentice-Hall)

Carone, L., Keppens, R., \& Decin, L. 2014, MNRAS, 445, 930

Carone, L., Keppens, R., \& Decin, L. 2015, MNRAS, 453, 2412

Carone, L., Keppens, R., \& Decin, L. 2016, MNRAS, 461, 1981

Charnay, B., Meadows, V. S., Misra, A., et al. 2015, ApJL, 813, L1

Clough, S. A., Kneizys, F. X., \& Davies, R. W. 1989, AtmRe, 23, 229

Clough, S. A., Shephard, M. W., Mlawer, E. J., et al. 2005, JQSRT, 91, 233

Dobrovolskis, A. R. 2009, Icar, 204, 1

Dressing, C., \& Charbonneau, D. 2015, ApJ, 807, 45

Foreman-Mackey, D., Hogg, D. W., \& Morton, T. D. 2014, ApJ, 795, 64

Fuji, Y., Del Genio, A., \& Amundsen, D. 2017, arXiv:1704.05878 
Gillon, M., Triaud, A. H. M. J., Demory, B. O., et al. 2017, Natur, 542, 456 Godolt, M., Grenfell, J. L., Hamann-Reinus, A., et al. 2015, P\&SS, 111, 62

Goldblatt, C., Robinson, T. D., Zahnle, K., \& Crisp, D. 2013, NatGe, 6, 661

Greene, T. P., Line, M. R., Cezar, M., et al. 2016, ApJ, 817, 17

Grimm, S., \& Heng, K. 2015, ApJ, 808, 182

Haqq-Misra, J., \& Kopparapu, R. K. 2015, MNRAS, 446, 428

Hack, J. J. 1994, JGR, 99, 5551

Haqq-Misra, J., Kopparapu, R. K., \& Batalha, N. 2016, ApJ, 827, 120

Hill, P. G., Manners, J., \& Petch, J. C. 2015, QJRMS, 137, 219

Hunke, E. C., \& Lipscomb, W. H. 2008, T-3 Fluid Dynamics Group, Los Alamos National Laboratory, Tech. Rep. LA-CC-06-012.

Hunten, D. M. 1973, JAS, 30, 1481

Joshi, M. M., Haberle, R. M., \& Reynolds, R. T. 1997, Icar, 129, 450

Kane, S. R., Hill, M. L., Kasting, J. F., et al. 2016, ApJ, 830, 1

Kasting, J. F., Chen, H., \& Kopparapu, R. K. 2015, ApJL, 813, 1

Kasting, J. F., Kopparapu, R. K., Ramirez, R., et al. 2014, PNAS, 111, 12641

Kasting, J. F., Whitmire, D. P., \& Reynolds, R. T. 1993, Icar, 101, 108

Kodama, T., Genda, H., Abe, Y., et al. 2015, ApJ, 812, 165

Kopparapu, R. K., Ramirez, R., Kasting, J. F., et al. 2013, ApJ, 765, 131

Kopparapu, R. K., Ramirez, R. M., SchottelKotte, J., et al. 2014, ApJL, 787, L29

Kopparapu, R. K., Wolf, E. T., Haqq-Misra, J., et al. 2016, ApJ, 819,

Larson, E. J. L., Toon, O. B., \& Friedson, A. J. 2014, Icar, 243, 400

Lauritzen, P. H., Bacmeister, J. T., Dubos, T., et al. 2014, JAMES, 6, 129

Lebonnois, S., Covey, C., Grossman, A., et al. 2012, JGR, 117, E12004

Leconte, J., Forget, F., Charnay, B., et al. 2013a, A\&A, 554, A69

Leconte, J., Forget, F., Charnay, B., Wordsworth, R., \& Pottier, A. 2013b, Natur, 504, 268

Leconte, J., Wu, H., Menou, K., \& Murray, N. 2015, Sci, 347, 632

Lin, S-J., \& Rood, R. B. 1996, MWRv, 124, 2046

Luger, R., \& Barnes, R. 2015, AsBio, 15, 119

Meadows, V. S., \& Crisp, D. 1996, JGR, 101, 4595

Meadows, V. S., Arney, G. N., \& Schwieterman, E. W. 2016, arXiv:1608. 08620

Merlis, T. M., \& Schneider, T. 2010, JAMES, 2, 13

Misra, A., Meadows, V. S., \& Crisp, D. 2014, ApJ, 792, 61

Nakajima, S., Hayashi, Y-Y., \& Abe, Y. 1992, JAtS, 49, 2256

Neale, R. B., Chen, C. C., Gettelman, A., et al. 2010, Description of the NCAR Community Atmosphere Model (CAM 5.0), NCAR/TN-486+STR NCAR Technical Note

Parish, H. F., Schubert, G., Covey, C., et al. 2011, Icar, 212, 42

Paynter, D. J., \& Ramaswamy, V. 2011, JGRD, 116, D20302

Petigura, E. A., Howard, A. W., \& Marcy, G. W. 2013, PNAS, 110, 19273
Pierrehumbert, R. T. 2010, Principles of Planetary Climate (Cambridge: Cambridge Univ. Press)

Pierrehumbert, R. T., \& Gaidos, E. 2011, ApJL, 734, L13

Pincus, R., Barker, H. W., \& Morcrette, J. 2003, JGRD, 108, 4376

Popp, M., Schmidt, H., \& Marotzke, J. 2016, NatCo, 7, 10627

Pontoppidan, K. M., Pickering, T. E., Laidler, V. J., et al. 2016, Proc. SPIE, 9910, 991016

Ramirez, R. M., \& Kaltenegger, L. 2017, ApJL, 837, L4

Rasch, P., \& Kristjansson, J. 1998, JCli, 11, 1587

Raymond, D. J., \& Blyth, A. 1986, JAS, 43, 22

Raymond, D. .J., \& Blyth, A. 1992, JAS, 49, 21

Ribas, I., Bolmont, E., Selsis, F., et al. 2016, A\&A, 596, A111

Rogelj, J., Meinshausen, M., \& Knutti, R. 2012, NatCC, 2, 248

Robinson, T. D., Meadows, V. S., Crisp, D., et al. 2011, AsBio, 11, 393

Robinson, T. D., Maltagliati, L., Marley, M. S., et al. 2014, PNAS, 111, 9042

Rothman, L. S., Barbe, A., \& Benner, D. C. 2003, JQSRT, 82, 5

Salameh, J., Popp, M., \& Marotzke, J. 2017, CIDy, doi:10.1007/s00382-0173548-6

Schwieterman, E. W., Meadows, V. S., Domagal-Goldman, S., et al. 2016, ApJL, 819, L13

Selsis, F., Kasting, J. F., Levrard, B., et al. 2007, A\&A, 476, 1373

Sherwood, S. C., \& Huber, M. 2010, PNAS, 107, 9552

Shields, A. L., Meadows, V. S., Bitz, C. M., et al. 2013, AsBio, 13, 715

Silburt, A., Gaidos, E., \& Wu, Y. 2015, ApJ, 799, 180

Tian, F., \& Ida, S. 2015, NatGe, 8, 177

Toon, B., McKay, C., Ackerman, T., et al. 1989, JGR, 94, 16287

Turbet, M., Leconte, J., Selsis, F., et al. 2016, A\&A, 596, A112

Wang, Y., Liu, Y., Tian, F., et al. 2016, ApJL, 823, L20

Way, M. J., Del Genio, A. D., Kelley, M., Aleinov, I., \& Clune, T. 2015, arXiv: 1511.07283

Way, M. J., Del Genio, A. D., Kiang, N. Y., et al. 2016, GRL, 43, 8376

Way, M. J., \& Georgakarakos, N. 2017, ApJL, 835, L1

Wolf, E., \& Toon, B. 2013, AsBio, 13, 1

Wolf, E., \& Toon, O. B. 2014, GeoRL, 41, 167

Wolf, E., \& Toon, O. B. 2015, JGRD, 120, 5775

Wordsworth, R., \& Pierrehumbert, R. 2014, ApJL, 785, L20

Wordsworth, R., Forget, F., \& Eyment, V. 2010, Icar, 210, 992

Yang, J., Cowan, N. B., \& Abbot, D. S. 2013, ApJL, 771, L45

Yang, J., Gwenael, B., Fabrycky, D., \& Abbot, D. 2014a, ApJL, 787, L2

Yang, J., Leconte, J., Wolf, E. T., et al. 2016, ApJ, 826, 222

Zhang, G., \& McFarlane, N. 1995, AtO, 33, 3

Zsom, A., Seager, S., de Wit, J., et al. 2013, ApJ, 778, 109 